\title{
Two Person Zero-sum Game in Weak Formulation and Path Dependent Bellman-Isaacs Equation
}

\author{
Triet PHAM $^{*} \quad$ Jianfeng $\mathrm{ZHANG}^{\dagger \ddagger}$
}

September 19, 2018

\begin{abstract}
In this paper we study a two person zero sum stochastic differential game in weak formulation. Unlike standard literature which uses strategy type of controls, the weak formulation allows us to consider the game with control against control. We shall prove the existence of game value under natural conditions. Another main feature of the paper is that we allow for non-Markovian structure, and thus the game value is a random process. We characterize the value process as the unique viscosity solution of the corresponding path dependent Bellman-Isaacs equation, a notion recently introduced by Ekren, Keller, Touzi and Zhang [14] and Ekren, Touzi and Zhang [15, 16, 17.
\end{abstract}

Key words: Zero sum games, weak formulation, Path dependent PDEs, viscosity solutions, Dynamic programming principles.

AMS 2000 subject classifications: 91A15, 60H30, 35D40, 35K10.

\section{Introduction}

Since the seminal paper Fleming and Souganidis [19], two person zero sum stochastic differential games have been studied extensively in the literature, see e.g. [1, [2], 3], 4], 5], 7], [13, 18], 21], 22], 23], 27], 34], to mention a few. There are typically two approaches. One is to use the viscosity theory, namely to show that the value function of the game is

\footnotetext{
*University of Southern California, Department of Mathematics, trietpha@usc.edu. Research supported by USC Graduate School Dissertation Completion Fellowship.

${ }^{\dagger}$ University of Southern California, Department of Mathematics, jianfenz@usc.edu. Research supported in part by NSF grant DMS 10-08873. Part of the research was done while this author was visiting Shandong University, whose hospitality is greatly appreciated.

${ }^{\ddagger}$ The authors would like to thank Rainer Buckdahn and Lihe Wang for very helpful discussion. In particular we thank Buckdahn for providing us the counterexample Example 8.1 and Wang for showing us the proof of Lemma 6.3
} 
the unique viscosity solution of the associated Bellman-Isaacs equation, and the other is to use the Backward SDE approach, which characterizes the value process as the solution to a related BSDE.

To be precise, let $u$ and $v$ denote the controls of the two players, $B$ a Brownian motion, $X^{S, u, v}$ the controlled state process in the strong formulation:

$$
X_{t}^{S, u, v}=x+\int_{0}^{t} b\left(s, u_{s}, v_{s}\right) d s+\int_{0}^{t} \sigma\left(s, u_{s}, v_{s}\right) d B_{s},
$$

and $J(u, v)$ the corresponding value (utility or cost) which is determined by $X^{S, u, v}, B$, and $(u, v)$. The lower and upper values of the game are defined as:

$$
\underline{V}_{0}:=\sup _{u \in \mathcal{U}} \inf _{v \in \mathcal{V}} J(u, v), \quad \bar{V}_{0}:=\inf _{v \in \mathcal{V}} \sup _{u \in \mathcal{U}} J(u, v),
$$

where $\mathcal{U}$ and $\mathcal{V}$ are appropriate sets of admissible controls. It is clear that $\underline{V}_{0} \leq \bar{V}_{0}$. Two central problems in the game literature are:

(i) When does the game value exists, namely $V_{0}:=\underline{V}_{0}=\bar{V}_{0}$ ?

(ii) Given the existence of the game value, is there a saddle point? That is, we want to find $\left(u^{*}, v^{*}\right) \in \mathcal{U} \times \mathcal{V}$ such that $V_{0}=J\left(u^{*}, v^{*}\right)=\inf _{v \in \mathcal{V}} J\left(u^{*}, v\right)=\sup _{u \in \mathcal{U}} J\left(u, v^{*}\right)$.

However, even under reasonable assumptions, the game value may not exist. We shall provide a counterexample, see Example 8.1 below, which is due to Buckdahn.

To overcome the difficulty, Fleming and Souganidis [19] introduced strategy types of controls:

$$
\underline{V}_{0}^{\prime}:=\sup _{\alpha \in \mathcal{A}} \inf _{v \in \mathcal{V}} J(\alpha(v), v), \quad \bar{V}_{0}^{\prime}:=\inf _{\beta \in \mathcal{B}} \sup _{u \in \mathcal{U}} J(u, \beta(u)),
$$

Here $\alpha: \mathcal{V} \rightarrow \mathcal{U}$ and $\beta: \mathcal{U} \rightarrow \mathcal{V}$ are so called strategies and $\mathcal{A}, \mathcal{B}$ are appropriate sets of admissible strategies. Under the Isaacs condition and assuming the comparison principle for the viscosity solution of the corresponding Bellman-Isaacs equation holds, [19] showed that $\underline{V}_{0}^{\prime}=\bar{V}_{0}^{\prime}$. This work has been extended by many authors in various aspects. In particular, Buckdahn and Li [5] defined $J(u, v)$ via Backward SDEs, and very recently Bayraktar and Yao [1] used doubly reflected BSDEs. The main drawback of this approach, however, is that the two players have non-symmetric information, and for $\underline{V}_{0}^{\prime}$ and $\bar{V}_{0}^{\prime}$, the roles of two players are switched. Consequently, it is less convenient to study the saddle point in this setting.

We propose to attack the problem in weak formulation, which is more convenient for proving the Dynamic Programming Principle. Note that in (1.1) the controls $(u, v)$ actually mean $u(B),. v(B$.$) . Our weak formulation is equivalent to the following feedback type of$ controls:

$$
X_{t}^{W, u, v}=x+\int_{0}^{t} b\left(s, u_{s}\left(X^{W, u, v}\right), v_{s}\left(X^{W, u, v}\right)\right) d s+\int_{0}^{t} \sigma\left(s, u_{s}\left(X^{W, u, v}\right), v_{s}\left(X^{W, u, v}\right)\right) d B_{s},(1.2
$$


Here $X^{W, u, v}$ denotes the path of $X^{W, u, v}$ and the superscript ${ }^{W}$ stands for weak formulation. Under natural assumptions, we show that the game value does exist. The advantage of the weak formulation setting is that we are using control against control, thus one can define the saddle point naturally. When there is only drift control, namely $\sigma$ is independent of $(u, v)$, one can prove the existence of saddle point under mild conditions. However, when there is diffusion control, the problem is much more involved. We shall obtain some approximate saddle point.

We remark that, when there is only drift control, the weak formulation has already been used in the literature, see Bensoussan and Lions [2] for Markovian case and Hamadene and Lepetier [21] for non-Markovian case. The former one relies on PDE arguments and the latter one uses Backward SDEs. The advantage in this case is that one can easily obtain the weak solution of SDE (1.2) by applying the Girsanov Theorem. Our general case with diffusion control has different nature. Roughly speaking, the drift control is associated with semi-linear PDEs, while the diffusion control is associate with fully nonlinear PDEs. We also note that, in a Markovian model but also with optimal stopping problem, Karatzas and Sudderth [23] studied the game problem with diffusion control in weak formulation, under certain strong conditions.

Another main feature of our paper is that we study the game in non-Markovian framework, or say in a path dependent manner. The standard approach in the literature, e.g. [19] and [5], is to prove that the lower value and the upper value are a viscosity solution (or viscosity semi-solution) of the corresponding Bellman-Isaacs equation, then by assuming the comparison principle for the viscosity solution of the PDE, one obtains the existence of the game value. These works rely on the PDE arguments and thus works only in Markovian setting. In a series of papers, Ekren, Keller, Touzi and Zhang [14] and Ekren, Touzi and Zhang [15, 16, 17] introduced a notion of viscosity solution for the so called path dependent PDEs and established its wellposedness. This enables us to extend the above approach to path dependent setting. Indeed, based on the dynamic programming principle we establish, we show that the lower value and the upper value of the game are viscosity solutions of the corresponding path dependent Bellman-Isaacs equations. Then, under the Isaacs condition and assuming the uniqueness of viscosity solutions, we characterize the game value as the unique viscosity solution of the path dependent Bellman-Isaacs equation.

Finally we remark that, due to weak formulation with diffusion control, this paper is by nature closely related to the second order BSDEs (2BSDEs, for short) introduced by Cheridito, Soner, Touzi and Vicoir [8] and Soner, Touzi and Zhang [31, 32], and the Gexpectation introduced by Peng [29]. While more involved here, our arguments for Dynamic Programming Principle follow the idea in [31, 32] and Peng [28]. However, $G$-expectations and 2BSDEs involve only stochastic optimization and thus the generator is convex in terms 
of the hessian. Consequently, the dynamic value process is a supermartingale under each associated probability measure. For our game problem, the Bellman-Isaacs equation is nonconvex, and the value process is not a supermartingale anymore. Under additional technical conditions, we conjecture that our value process will be a semi-martingale. This requires to develop a semi-martingale theory under nonlinear expectation and to generalize the 2BSDE theory to non-convex generators. We established some norm estimates for semi-martingales in another paper Pham and Zhang [30] and will leave the general 2BSDE theory for future research.

The rest of the paper is organized as follows. In Section 2 we present some preliminaries. The game problem is introduced in Section 3. In Sections 4 and 5 we prove the dynamic programming principle and the viscosity property, respectively. In Section 6 we study the comparison principle for PPDEs and in Section 7 we investigate approximate saddle points. Finally some technical proofs are presented Appendix.

\section{Preliminaries}

\subsection{The canonical space}

Let $\Omega:=\left\{\omega \in C\left([0, T], \mathbb{R}^{d}\right): \omega_{0}=\mathbf{0}\right\}$, the set of continuous paths starting from the origin, $B$ the canonical process, $\mathbb{F}$ the filtration generated by $B, \mathbb{P}_{0}$ the Wiener measure, and $\Lambda:=[0, T] \times \Omega$. Here and in the sequel, for notational simplicity we use $\mathbf{0}$ to denote vectors or matrices with appropriate dimensions whose components are all equal to 0 . Let $\mathbb{S}^{d}$ denote the set of $d \times d$ matrices, $\mathbb{S}_{\geq \mathbf{0}}^{d}:=\left\{\sigma \in \mathbb{S}^{d}: \sigma \geq \mathbf{0}\right\}$, and

$$
x \cdot x^{\prime}:=\sum_{i=1}^{d} x_{i} x_{i}^{\prime} \text { for any } x, x^{\prime} \in \mathbb{R}^{d}, \quad \gamma: \gamma^{\prime}:=\operatorname{Trace}\left[\gamma \gamma^{\prime}\right] \text { for any } \gamma, \gamma^{\prime} \in \mathbb{S}^{d} .
$$

We define a norm on $\Omega$ and a metric on $\Lambda$ as follows: for any $(t, \omega),\left(t^{\prime}, \omega^{\prime}\right) \in \Lambda$,

$$
\|\omega\|_{t}:=\sup _{0 \leq s \leq t}\left|\omega_{s}\right|, \quad \mathbf{d}_{\infty}\left((t, \omega),\left(t^{\prime}, \omega^{\prime}\right)\right):=\left|t-t^{\prime}\right|+\left\|\omega . \wedge t-\omega_{. \wedge t^{\prime}}^{\prime}\right\|_{T} .
$$

Then $\left(\Omega,\|\cdot\|_{T}\right)$ and $\left(\Lambda, \mathbf{d}_{\infty}\right)$ are complete metric spaces.

Definition 2.1 Let $Y: \Lambda \rightarrow \mathbb{R}$ be an $\mathbb{F}$-progressively measurable process.

(i) We say $Y \in \mathbb{L}^{\infty}(\Lambda)$ if $Y$ is bounded.

(ii) We say $Y \in C^{0}(\Lambda)$ (resp. $U C(\Lambda)$ ) if $Y$ is continuous (resp. uniformly continuous) in $(t, \omega)$. Moreover, we denote $C_{b}^{0}(\Lambda):=C^{0}(\Lambda) \cap \mathbb{L}^{\infty}(\Lambda)$ and $U C_{b}(\Lambda):=U C(\Lambda) \cap \mathbb{L}^{\infty}(\Lambda)$.

(iii) We say $Y \in \underline{\mathcal{U}}$ if $Y$ is bounded from above, upper semi-continuous (u.s.c. for short) from right in $t$, and there exists a modulus of continuity function $\rho$ such that for $(t, \omega),\left(t^{\prime}, \omega^{\prime}\right) \in \Lambda$ :

$$
Y(t, \omega)-Y\left(t^{\prime}, \omega^{\prime}\right) \leq \rho\left(\mathbf{d}_{\infty}\left((t, \omega),\left(t^{\prime}, \omega^{\prime}\right)\right)\right) \text { whenever } t \leq t^{\prime},
$$

and we say $Y \in \overline{\mathcal{U}}$ if $-Y \in \underline{\mathcal{U}}$. 
It is clear that $\underline{\mathcal{U}} \cap \overline{\mathcal{U}}=U C_{b}(\Lambda)$. Moreover, we denote by $\mathbb{L}^{\infty}\left(\Lambda, \mathbb{R}^{d}\right)$ the space of $\mathbb{R}^{d}$ valued processes whose components are in $\mathbb{L}^{\infty}(\Lambda)$, and define other similar notations in the same spirit.

We next introduce the shifted spaces. Let $0 \leq s \leq t \leq T$.

- Let $\Omega^{t}:=\left\{\omega \in C\left([t, T], \mathbb{R}^{d}\right): \omega_{t}=\mathbf{0}\right\}$ be the shifted canonical space; $B^{t}$ the shifted canonical process on $\Omega^{t} ; \mathbb{F}^{t}$ the shifted filtration generated by $B^{t}, \mathbb{P}_{0}^{t}$ the Wiener measure on $\Omega^{t}$, and $\Lambda^{t}:=[t, T] \times \Omega^{t}$.

- Define $\|\cdot\|_{s}$ on $\Omega^{t}, \mathbf{d}_{\infty}$ on $\Lambda^{t} \times \Lambda^{t}$, and $C^{0}\left(\Lambda^{t}\right)$ etc. in the spirit of (2.3) and Definition 2.1

- For $\omega \in \Omega^{s}$ and $\omega^{\prime} \in \Omega^{t}$, define the concatenation path $\omega \otimes_{t} \omega^{\prime} \in \Omega^{s}$ by:

$$
\left(\omega \otimes_{t} \omega^{\prime}\right)(r):=\omega_{r} \mathbf{1}_{[s, t)}(r)+\left(\omega_{t}+\hat{\omega}_{r}^{\prime}\right) \mathbf{1}_{[t, T]}(r), \text { for all } r \in[s, T] .
$$

- Let $s \in[0, T)$ and $\omega \in \Omega^{s}$. For an $\mathcal{F}_{T}^{s}$-measurable random variable $\xi$, an $\mathbb{F}^{s}$ progressively measurable process $X$ on $\Omega^{s}$, and $t \in(s, T]$, define the shifted $\mathcal{F}_{T}^{t}$-measurable random variable $\xi^{t, \omega}$ and $\mathbb{F}^{t}$-progressively measurable process $X^{t, \omega}$ on $\Omega^{t}$ by:

$$
\xi^{t, \omega}\left(\omega^{\prime}\right):=\xi\left(\omega \otimes_{t} \omega^{\prime}\right), \quad X^{t, \omega}\left(\omega^{\prime}\right):=X\left(\omega \otimes_{t} \omega^{\prime}\right), \quad \text { for all } \quad \omega^{\prime} \in \Omega^{t} .
$$

It is clear that, for any $(t, \omega) \in \Lambda$ and any $Y \in \mathbb{L}^{\infty}(\Lambda)$, we have $Y^{t, \omega} \in \mathbb{L}^{\infty}\left(\Lambda^{t}\right)$. Similarly the property holds for other spaces defined in Definition 2.1.

\subsection{Probability measures}

In this subsection we introduce the probability measures on $\Omega^{t}$ in different formulations. First, let $\sigma \in \mathbb{L}^{\infty}\left(\Lambda, \mathbb{S}_{\geq \mathbf{0}}^{d}\right), b \in \mathbb{L}^{\infty}\left(\Lambda, \mathbb{R}^{d}\right)$. Define

$$
\mathbb{P}^{S, \sigma, b}:=\mathbb{P}_{0} \circ\left(X^{S, \sigma, b}\right)^{-1} \quad \text { where } \quad X_{t}^{S, \sigma, b}:=\int_{0}^{t} b_{s} d s+\int_{0}^{t} \sigma_{s} d B_{s}, \quad \mathbb{P}_{0} \text {-a.s. }
$$

Here the superscript ${ }^{S}$ stands for strong formulation. We next introduce the corresponding weak formulation. We denote a probability measure $\mathbb{P}$ on $\Omega$ as $\mathbb{P}^{W, \sigma, b}$ if

$$
M_{t}^{b}:=B_{t}-\int_{0}^{t} b_{s} d s \text { is a } \mathbb{P} \text {-martingale and }\left\langle M^{b}\right\rangle_{t}=\int_{0}^{t} \sigma_{s}^{2} d s \mathbb{P} \text {-a.s. }
$$

Here the quadratic variation $\left\langle M^{b}\right\rangle$ is under $\mathbb{P}$. We remark that $\mathbb{P}^{W, \sigma, b}:=\mathbb{P}_{0} \circ\left(X^{W, \sigma, b}\right)^{-1}$, where $X^{W, \sigma, b}$ is a weak solution of the following SDE (with random measurable coefficients):

$$
X_{t}^{W, \sigma, b}:=\int_{0}^{t} b_{s}\left(X^{W, \sigma, b}\right) d s+\int_{0}^{t} \sigma_{s}\left(X^{W, \sigma, b}\right) d B_{s}, \quad \mathbb{P}_{0} \text {-a.s. }
$$

In other words, we are considering feedback type of controls. 
In this paper we shall use the weak formulation, which is more convenient for proving Dynamical Programming Principle. We note that, for arbitrarily given $(\sigma, b)$, the SDE (2.7) may not have a weak solution, namely there is no $\mathbb{P}$ such that $\mathbb{P}=\mathbb{P}^{W, \sigma, b}$. Let

$$
\begin{aligned}
& \bar{\Xi}^{W}:=\left\{(\sigma, b) \in \mathbb{L}^{\infty}\left(\Lambda, \mathbb{S}_{\geq \mathbf{0}}^{d}\right) \times \mathbb{L}^{\infty}\left(\Lambda, \mathbb{R}^{d}\right): \operatorname{SDE}(2.7) \text { has a unique weak solution }\right\} \\
& \bar{\Xi}^{S}:=\left\{(\sigma, b) \in \mathbb{L}^{\infty}\left(\Lambda, \mathbb{S}_{\geq \mathbf{0}}^{d}\right) \times \mathbb{L}^{\infty}\left(\Lambda, \mathbb{R}^{d}\right): \operatorname{SDE}(2.7) \text { has a unique strong solution }\right\} .
\end{aligned}
$$

For probability measures on the shifted space $\Omega^{t}$, we define $\mathbb{P}^{S, t, \sigma, b}, \mathbb{P}^{W, t, \sigma, b}$, and $\bar{\Xi}^{W, t}, \bar{\Xi}^{S, t}$, etc. similarly.

We next introduce the regular conditional probability distribution (r.c.p.d for short) due to Stroock and Varadhan [33]. We shall follow the presentation in Soner, Touzi and Zhang [31. Let $\mathbb{P}$ be an arbitrary probability measure on $\Omega$ and $\tau$ be an $\mathbb{F}$ - stopping time. The r.c.p.d. $\left\{\mathbb{P}^{\tau, \omega}, \omega \in \Omega\right\}$ satisfies:

- For each $\omega, \mathbb{P}^{\tau, \omega}$ is a probability measure on $\mathcal{F}_{T}^{\tau(\omega)}$;

- For every bounded $\mathcal{F}_{T}$-measurable random variable $\xi$ :

$$
\mathbb{E}^{\mathbb{P}}\left[\xi \mid \mathcal{F}_{\tau}\right](\omega)=\mathbb{E}^{\mathbb{P}^{\tau, \omega}}\left[\xi^{\tau(\omega), \omega}\right], \quad \mathbb{P} \text {-a.s. }
$$

The following simple lemma will be important for the proof of Dynamic Programming Principle in Section 4 below. Its proof is postponed to Appendix.

Lemma 2.2 Let $(\sigma, b) \in \bar{\Xi}^{S}$ (resp. $\left.\bar{\Xi}^{W}\right), t \in[0, T],\left\{E_{i}, 1 \leq i \leq n\right\} \subset \mathcal{F}_{t}$ be a partition of $\Omega$, and $\left(\sigma^{i}, b^{i}\right) \in \bar{\Xi}^{S, t}\left(\right.$ resp. $\left.\bar{\Xi}^{W, t}\right)$. Define

$$
\bar{\sigma}(\omega):=\sigma(\omega) \mathbf{1}_{[0, t)}+\sum_{i=1}^{n} \sigma^{i}\left(\omega^{t}\right) \mathbf{1}_{E^{i}} \mathbf{1}_{[t, T]}, \quad \bar{b}(\omega):=b(\omega) \mathbf{1}_{[0, t)}+\sum_{i=1}^{n} b^{i}\left(\omega^{t}\right) \mathbf{1}_{E^{i}} \mathbf{1}_{[t, T]} .
$$

Then $(\bar{\sigma}, \bar{b}) \in \bar{\Xi}^{S}$ (resp. $\bar{\Xi}^{W}$ ), and, for $i=1, \cdots, n$,

$$
\mathbb{P}^{\bar{\sigma}, \bar{b}}=\mathbb{P}^{\sigma, b} \text { on } \mathcal{F}_{t} \quad \text { and } \quad\left(\mathbb{P}^{\bar{\sigma}, \bar{b}}\right)^{t, \omega}=\mathbb{P}^{t, \sigma^{i}, b^{i}} \text { for } \mathbb{P}^{\sigma, b} \text {-a.e. } \omega \in E_{i} .
$$

\subsection{Viscosity solutions of path dependent PDEs}

Our notion of viscosity solutions of Path Dependent PDEs (PPDEs for short) is introduced by Ekren, Keller, Touzi and Zhang [14] for semilinear PPDE and Ekren, Touzi and Zhang [15, 16] for fully nonlinear PPDE. We follow the presentation in [15, 16] here.

For any constant $L>0$, denote

$$
\mathcal{P}_{L}^{t}:=\left\{\mathbb{P}^{W, t, \sigma, b}:|b| \leq L, \mathbf{0} \leq \sigma \leq \sqrt{2 L} I_{d}\right\}, \quad \text { and } \quad \mathcal{P}_{\infty}^{t}:=\cup_{L>0} \mathcal{P}_{L}^{t} .
$$

We remark that in $\mathcal{P}_{L}^{t}$ we do not require the uniqueness of weak solution. 
Let $Y \in C^{0}(\Lambda)$. For $t \in[0, T)$, we define the right time-derivative, if it exists, as in Dupire [12] and Cont and Fournie [9]:

$$
\partial_{t} Y(t, \omega):=\lim _{h \downarrow 0} \frac{1}{h}[Y(t+h, \omega \cdot \wedge t)-Y(t, \omega)] .
$$

For the final time $T$, we define, whenever the following limit exists:

$$
\partial_{t} Y(T, \omega):=\lim _{t \uparrow T} \partial_{t} Y(t, \omega)
$$

Definition 2.3 (i) We say $Y \in C^{1,2}(\Lambda)$ if $Y \in C^{0}(\Lambda), \partial_{t} Y \in C^{0}(\Lambda)$, and there exist $\partial_{\omega} Y \in C^{0}\left(\Lambda, \mathbb{R}^{d}\right), \partial_{\omega \omega}^{2} Y \in C^{0}\left(\Lambda, \mathbb{S}^{d}\right)$ such that, for any $(s, \omega) \in[0, T) \times \Omega$ and any $\mathbb{P} \in \mathcal{P}_{\infty}^{s}$, $Y^{s, \omega}$ is a local $\mathbb{P}$-semimartingale and it holds:

$$
d Y_{t}^{s, \omega}=\left(\partial_{t} Y_{t}\right)^{s, \omega} d t+\left(\partial_{\omega} Y_{t}\right)^{s, \omega} \cdot d B_{t}^{s}+\frac{1}{2}\left(\partial_{\omega \omega}^{2} Y_{t}\right)^{s, \omega}: d\left\langle B^{s}\right\rangle_{t}, \quad \mathbb{P}-a . s .
$$

(ii) We say $Y \in C_{b}^{1,2}(\Lambda)$ if $Y \in U C_{b}(\Lambda), \partial_{t} Y \in C_{b}^{0}(\Lambda)$, and the above $\partial_{\omega} Y$ and $\partial_{\omega \omega}^{2} Y$ exist and are in $C_{b}^{0}\left(\Lambda, \mathbb{R}^{d}\right)$ and $C_{b}^{0}\left(\Lambda, \mathbb{S}^{d}\right)$, respectively.

Next, let $\mathcal{T}$ denote the set of $\mathbb{F}$-stopping times, and $\mathcal{H} \subset \mathcal{T}$ the subset of those hitting times $\mathrm{H}$ taking the following form: for some open and convex set $O \subset \mathbb{R}^{d}$ containing $\mathbf{0}$ and some $0<t_{0} \leq T$,

$$
\mathrm{H}:=\inf \left\{t: B_{t} \in O^{c}\right\} \wedge t_{0}=\inf \left\{t: d\left(\omega_{t}, O^{c}\right)=0\right\} \wedge t_{0}
$$

We may define $C^{1,2}\left(\Lambda^{t}\right), C_{b}^{1,2}\left(\Lambda^{t}\right), \mathcal{T}^{t}$, and $\mathcal{H}^{t}$ similarly. It is clear that, for any $(t, \omega)$ and $Y \in C^{1,2}(\Lambda)$ (resp. $Y \in C_{b}^{1,2}(\Lambda)$ ), we have $Y^{t, \omega} \in C^{1,2}\left(\Lambda^{t}\right)\left(\operatorname{resp} . Y^{t, \omega} \in C_{b}^{1,2}(\Lambda)\right.$ ), and for any $\mathrm{H} \in \mathcal{H}$ such that $\mathrm{H}(\omega)>t$, we have $\mathrm{H}^{t, \omega} \in \mathcal{H}^{t}$.

For any $L>0,(t, \omega) \in \Lambda$ with $t<T$, and $\mathbb{F}$-adapted process $Y$, define

$$
\begin{aligned}
\underline{\mathcal{A}}^{L} Y(t, \omega):= & \left\{\varphi \in C_{b}^{1,2}\left(\Lambda^{t}\right): \text { for some } \mathrm{H} \in \mathcal{H}^{t},\right. \\
& \left.\left(\varphi-Y^{t, \omega}\right)(t, \mathbf{0})=\inf _{\tau \in \mathcal{T}^{t}} \inf _{\mathbb{P} \in \mathcal{P}_{L}^{t}} \mathbb{E}^{\mathbb{P}}\left[\left(\varphi-Y^{t, \omega}\right)_{\tau \wedge \mathrm{H}}\right]\right\}, \\
\overline{\mathcal{A}}^{L} Y(t, \omega):= & \left\{\varphi \in C_{b}^{1,2}\left(\Lambda^{t}\right): \text { for some } \mathrm{H} \in \mathcal{H}^{t},\right. \\
& \left.\left(\varphi-Y^{t, \omega}\right)(t, \mathbf{0})=\sup _{\tau \in \mathcal{T}^{t}} \sup _{\mathbb{P} \in \mathcal{P}_{L}^{t}} \mathbb{E}^{\mathbb{P}}\left[\left(\varphi-Y^{t, \omega}\right)_{\tau \wedge \mathrm{H}}\right]\right\} .
\end{aligned}
$$

We are now ready to introduce the viscosity solution of PPDEs. Consider the following PPDE with generator $G$ :

$$
-\partial_{t} Y_{t}-G\left(t, \omega, Y_{t}, \partial_{\omega} Y_{t}, \partial_{\omega \omega}^{2} Y_{t}\right)=0
$$


Definition 2.4 (i) Let $L>0$. We say $Y \in \underline{\mathcal{U}}$ (resp. $\overline{\mathcal{U}}$ ) is a viscosity L-subsolution (resp. $L$-supersolution) of PPDE (2.16) if, for any $(t, \omega) \in[0, T) \times \Omega$ and any $\varphi \in \underline{\mathcal{A}}^{L} Y(t, \omega)$ (resp. $\left.\varphi \in \overline{\mathcal{A}}^{L} Y(t, \omega)\right)$ :

$$
\left(-\partial_{t} \varphi-G^{t, \omega}\left(., Y^{t, \omega}, \partial_{\omega} \varphi, \partial_{\omega \omega}^{2} \varphi\right)\right)(t, \mathbf{0}) \leq(\text { resp. } \geq) 0 .
$$

(ii) We say $Y \in \underline{\mathcal{U}}$ (resp. $\overline{\mathcal{U}}$ ) is a viscosity subsolution (resp. supersolution) of PPDE (2.16) if $Y$ is viscosity $L$-subsolution (resp. L-supersolution) of PPDE (2.16) for some $L>0$.

(iii) We say $Y \in U C_{b}(\Lambda)$ is a viscosity solution of PPDE (2.16) if it is both a viscosity subsolution and a viscosity supersolution.

Remark 2.5 For $0<L_{1}<L_{2}$, obviously $\mathcal{P}_{L_{1}}^{t} \subseteq \mathcal{P}_{L_{2}}^{t}$ and $\underline{\mathcal{A}}^{L_{2}} Y(t, \omega) \subseteq \underline{\mathcal{A}}^{L_{1}} Y(t, \omega)$. Then one can easily check that a viscosity $L_{1}$-subsolution must be a viscosity $L_{2}$-subsolution. Consequently, $Y$ is a viscosity solution of PPDE (2.16) iff there exists an $L \geq 1$ such that for all $\tilde{L} \geq L, Y$ is a viscosity $\tilde{L}$-subsolution. However, we require the same $L$ for all $(t, \omega)$. A similar statement holds for the viscosity supersolution.

Remark 2.6 (i) In the Markovian case, namely $Y(t, \omega)=Y\left(t, \omega_{t}\right)$ and $G=g\left(t, \omega_{t}, y, z, \gamma\right)$, our definition of viscosity solution is stronger than the standard viscosity solution in PDE literature. That is, $Y$ is a viscosity solution in our sense implies it is a viscosity solution in the standard sense as in Crandall, Ishii, and Lions [10].

(ii) The state space $\Lambda$ of PPDEs is not locally compact, and thus the standard arguments by using Ishii's lemma do not work in path dependent case. The main idea of [14, 15, 16] is to transform the definition to an optimal stopping problem in (3.2), which helps to obtain the comparison and hence the uniqueness of viscosity solutions.

\section{The zero-sum game}

\subsection{The admissible controls}

Let $\mathbb{U}$ and $\mathbb{V}$ be two Borel measurable spaces equipped with some topology. From now on we shall fix two $\mathbb{F}$-progressively measurable mapping:

$$
\sigma:[0, T] \times \mathbb{U} \times \mathbb{V} \rightarrow \mathbb{S}_{\geq \mathbf{0}}^{d}, \quad b:[0, T] \times \mathbb{U} \times \mathbb{V} \rightarrow \mathbb{R}^{d} .
$$

We shall always assume

Assumption $3.1 \sigma$ and $b$ are bounded by a constant $C_{0}$. 
For $t \in[0, T]$, let $\overline{\mathcal{U}}_{t}$ (resp. $\overline{\mathcal{V}}_{t}$ ) denote the set of $\mathbb{U}$-valued (resp. $\mathbb{V}$-valued), $\mathbb{F}^{t_{-}}$ progressively measurable processes $u$ (resp. $v$ ) on $\Lambda^{t}$. Throughout the paper, when $(u, v) \in$ $\overline{\mathcal{U}}_{t} \times \overline{\mathcal{V}}_{t}$ is given, for any process $\varphi$ on $\Lambda^{t}$ with appropriate dimension, we denote

$$
\widehat{\varphi}_{s}:=\widehat{\varphi}_{s}^{t, u, v}:=\varphi_{s} \sigma\left(s, u_{s}, v_{s}\right)
$$

Define

$$
\begin{gathered}
\Xi_{t}:=\left\{(u, v) \in \overline{\mathcal{U}}_{t} \times \overline{\mathcal{V}}_{t}:(\sigma(\cdot, u, v), \widehat{b}(\cdot, u, v)) \in \overline{\mathcal{A}}^{S, t}\right\} \\
X^{t, u, v}:=X^{W, t, \sigma(u, v), \widehat{b}(u, v)}, \quad \mathbb{P}^{t, u, v}:=\mathbb{P}^{W, t, \sigma(u, v), \widehat{b}(u, v)}, \quad \text { for }(u, v) \in \Xi_{t} .
\end{gathered}
$$

We note that, from now on, the $\sigma, b$ in previous section will actually be $\sigma\left(t, u_{t}, v_{t}\right)$ and $\widehat{b}\left(t, u_{t}, v_{t}\right)$ for some $(u, v) \in \mathcal{A}_{0}$. In particular, for the convenience of studying the BSDE later, we are considering SDE in the form

$$
X_{s}^{t, u, v}=\int_{t}^{s} \sigma\left(r, u_{r}\left(X_{.}^{t, u, v}\right), v_{r}\left(X^{t, u, v}\right)\right)\left[d B_{r}^{t}+b\left(r, u_{r}\left(X_{.}^{t, u, v}\right), v_{r}\left(X^{t, u, v}\right)\right) d r\right], \quad \mathbb{P}_{0^{-}}^{t} \text {.s. }
$$

Moreover, one can easily check that there exists a $\mathbb{P}^{t, u, v}$-Brownian motion $W^{t, u, v}$ such that

$$
d B_{s}^{t}=\sigma\left(s, u_{s}, v_{s}\right)\left[d W_{s}^{t, u, v}+b\left(s, u_{s}, v_{s}\right) d s\right], \quad \mathbb{P}^{t, u, v} \text {-a.s. }
$$

To formulate the game problem, we shall restrict the controls to subsets $\mathcal{U}_{t} \subset \overline{\mathcal{U}}_{t}$ and $\mathcal{V}_{t} \subset \overline{\mathcal{V}}_{t}$ whose elements $u$ and $v$ take the following form:

$$
\begin{gathered}
u=\sum_{i=0}^{m-1} \sum_{j=1}^{n_{i}} u_{i j} \mathbf{1}_{E_{j}^{i}} \mathbf{1}_{\left[t_{i}, t_{i+1}\right)}, \quad v=\sum_{i=0}^{m-1} \sum_{j=1}^{n_{i}} v_{i j} \mathbf{1}_{E_{j}^{i}} \mathbf{1}_{\left[t_{i}, t_{i+1}\right)}, \text { where } \\
t=t_{0}<\cdots<t_{m}=T,\left\{E_{j}^{i}\right\}_{1 \leq j \leq n_{i}} \subset \mathcal{F}_{t_{i}}^{t} \text { is a partition, and } u_{i j}, v_{i j} \text { are constants. }
\end{gathered}
$$

It is clear that, for $u \in \overline{\mathcal{U}}_{t}$,

$$
u \in \mathcal{U}_{t} \text { if and only if } u \text { takes finitely many values. }
$$

We have the following simple lemma whose proof is provided in Appendix for completeness.

Lemma 3.2 (i) $\mathcal{U}_{0}$ is closed under pasting. That is, for $u \in \mathcal{U}_{0}, t \in[0, T], u^{i} \in \mathcal{U}_{t}$, $i=1, \cdots, n$, and disjoint $\left\{E_{i}, i=1, \cdots, n\right\} \subset \mathcal{F}_{t}$, the following $\bar{u}$ is also in $\mathcal{U}_{0}$ :

$$
\bar{u}:=u \mathbf{1}_{[0, t)}+\left[\sum_{i=1}^{n} u^{i}\left(\omega^{t}\right) \mathbf{1}_{E_{i}}+u \mathbf{1}_{\cap_{i=1}^{n} E_{i}^{c}}\right] \mathbf{1}_{[t, T]} .
$$

(ii) Under Assumption 3.1, it holds $\mathcal{U}_{t} \times \mathcal{V}_{t} \subset \Xi_{t}$.

Proof. In light of (3.6), (i) is obvious. To see (ii), we notice that any pair of constant processes $(u, v)$ is obviously in $\Xi_{t}$. Then (ii) follows from repeated use of Lemma 2.2 . 


\subsection{The Backward SDEs}

Let $f(t, \omega, y, z, u, v): \Lambda \times \mathbb{R} \times \mathbb{R}^{d} \times \mathbb{U} \times \mathbb{V} \rightarrow \mathbb{R}$ be an $\mathbb{F}$-progressively measurable nonlinear generator. Throughout the paper, we shall assume

Assumption 3.3 (i) $f(t, \omega, 0, \mathbf{0}, u, v)$ is bounded by a constant $C_{0}$, and uniformly continuous in $(t, \omega)$ with a modulus of continuity function $\rho_{0}$.

(ii) $f$ is uniformly Lipschitz in $(y, z)$ with a Lipschitz constant $L_{0}$.

Now for any $(t, \omega) \in \Lambda,(u, v) \in \mathcal{U}_{t} \times \mathcal{V}_{t}, \tau \in \mathcal{T}^{t}$, and $\mathcal{F}_{\tau}^{t}$-measurable terminal condition $\eta$, recall the notation (3.1) and consider the following BSDE on $[t, \tau]$ :

$$
\mathcal{Y}_{s}=\eta+\int_{s}^{\tau} f^{t, \omega}\left(r, B^{t}, \mathcal{Y}_{r}, \widehat{\mathcal{Z}}_{r}, u_{r}, v_{r}\right) d r-\int_{s}^{\tau} \mathcal{Z}_{r} d B_{r}^{t}, \mathbb{P}^{t, u, v} \text {-a.s. }
$$

We have the following simple lemma whose proof is presented in Appendix for completeness.

Lemma 3.4 Let Assumptions 3.1 and 3.3 (ii) hold, and

$$
I_{0}^{2}(t, \omega, u, v):=\mathbb{E}^{\mathbb{P}^{t, u, v}}\left[|\eta|^{2}+\int_{t}^{\tau}\left|f^{t, \omega}\left(s, B_{\cdot}^{t}, 0, \mathbf{0}, u_{s}, v_{s}\right)\right|^{2} d s\right]<\infty .
$$

Then BSDE (3.7) has a unique solution, denoted as $\left(\mathcal{Y}^{t, \omega, u, v}[\tau, \eta], \mathcal{Z}^{t, \omega, u, v}[\tau, \eta]\right)$, and there exists a constant $C$, depending only on $C_{0}, L_{0}, T$, and the dimension $d$, such that

$$
\mathbb{E}^{\mathbb{P}^{t, u, v}}\left[\sup _{t \leq s \leq \tau}\left|\mathcal{Y}_{s}^{t, \omega, u, v}[\tau, \eta]\right|^{2}+\int_{t}^{\tau}\left|\widehat{\mathcal{Z}}_{s}^{t, \omega, u, v}[\tau, \eta]\right|^{2} d s\right] \leq C I_{0}^{2}(t, \omega, u, v) .
$$

Moreover, if $\tau \leq t+\delta$, then

$$
\mathbb{E}^{\mathbb{P}^{t, u, v}}\left[\sup _{t \leq s \leq \tau}\left|\mathcal{Y}_{s}^{t, \omega, u, v}[\tau, \eta]\right|\right] \leq C\left(\mathbb{E}^{\mathbb{P}^{t, u, v}}\left[|\eta|^{2}\right]\right)^{\frac{1}{2}}+C \delta^{\frac{1}{2}} I_{0}(t, \omega, u, v) .
$$

Throughout the paper, we shall use the generic constant $C$ which depends only on $C_{0}, L_{0}$, $T$, and the dimension $d$, and may vary from line to line.

\subsection{The value processes}

We now fix an $\mathcal{F}_{T}$-measurable terminal condition $\xi$ and assume throughout the paper:

Assumption $3.5 \xi$ is bounded by a constant $C_{0}$, and is uniformly continuous in $\omega$ with a modulus of continuity function $\rho_{0}$.

We now define the lower value and upper value of the game as follows:

$$
\underline{Y}(t, \omega):=\sup _{u \in \mathcal{U}_{t}} \inf _{v \in \mathcal{V}_{t}} \mathcal{Y}_{t}^{t, \omega, u, v}\left[T, \xi^{t, \omega}\right] ; \quad \bar{Y}(t, \omega):=\inf _{v \in \mathcal{V}_{t}} \sup _{u \in \mathcal{U}_{t}} \mathcal{Y}_{t}^{t, \omega, u, v}\left[T, \xi^{t, \omega}\right]
$$


As a direct consequence of Lemma 3.4, we have

$$
-C \leq \underline{Y} \leq \bar{Y} \leq C
$$

When there is no confusion, we will simplify the notations:

$$
\left(\mathcal{Y}^{t, \omega, u, v}, \mathcal{Z}^{t, \omega, u, v}\right):=\left(\mathcal{Y}^{t, \omega, u, v}\left[T, \xi^{t, \omega}\right], \mathcal{Z}^{t, \omega, u, v}\left[T, \xi^{t, \omega}\right]\right)
$$

Our goal of this paper is to show, under certain additional assumptions, that $\underline{Y}=\bar{Y}$ and it is the unique viscosity solution of certain PPDE. See Theorem 5.1 below.

Remark 3.6 (i) In this paper we restrict our controls to $\mathcal{U}_{t} \times \mathcal{V}_{t} \subset \Xi_{t}$. We note that in general $\overline{\mathcal{U}}_{t} \times \overline{\mathcal{V}}_{t}$ is not in $\Xi_{t}$. We may study the following problem though:

$$
\underline{Y}^{\prime}(t, \omega):=\sup _{u \in \overline{\mathcal{U}}_{t}} \inf _{v \in \overline{\mathcal{V}}_{t}(u)} \mathcal{Y}_{t}^{t, \omega, u, v}, \quad \bar{Y}^{\prime}(t, \omega):=\inf _{v \in \overline{\mathcal{V}}_{t}} \sup _{u \in \overline{\mathcal{U}}_{t}(v)} \mathcal{Y}_{t}^{t, \omega, u, v}
$$

where

$$
\overline{\mathcal{V}}_{t}(u):=\left\{v \in \overline{\mathcal{V}}_{t}:(u, v) \in \Xi_{t}\right\}, \quad \overline{\mathcal{U}}_{t}(v):=\left\{u \in \overline{\mathcal{U}}_{t}:(u, v) \in \Xi_{t}\right\},
$$

and we take the convention that, for the empty set $\phi, \sup _{\phi}[\cdot]=\infty$ and $\inf _{\phi}[\cdot]=-\infty$. However, we will be able to prove only partial Dynamic Programming Principle in this formulation.

(ii) Another important constraint we impose is that $\sigma$ and $b$ are independent of $\omega$. When $\sigma$ and $b$ are random, given $(t, \omega) \in \Lambda$, the solution $X^{t, \omega, u, v}$ of SDE (3.3) and its distribution $\mathbb{P}^{t, \omega, u, v}$ will depend on $\omega$ as well. This has some subtle consequences, e.g. in Lemma 4.1 concerning the regularity of the value processes. The main difficulty is that we do not have a good stability result for feedback type of SDEs (3.3). We hope to address this issue in future research.

(iii) Note that we may get rid of the drift $b$ by using Girsanov transformation, so all our results hold true when $b$ is random, given that $\sigma$ is independent of $\omega$. However, to simplify the presentation we assume $b$ is independent of $\omega$ as well.

Remark 3.7 For each $(u, v) \in \mathcal{U}_{0} \times \mathcal{V}_{0}$, denote $\tilde{u}_{t}:=u_{t}\left(X^{u, v}\right)$ and $\tilde{v}_{t}:=v_{t}\left(X^{u, v}\right)$. Then $(\tilde{u}, \tilde{v}) \in \mathcal{U}_{0} \times \mathcal{V}_{0}$ and $\mathbb{P}^{u, v}=\mathbb{P}^{S, \tilde{u}, \tilde{v}}:=\mathbb{P}^{S, \sigma(\tilde{u}, \tilde{v}), \widehat{b}(\tilde{u}, \tilde{v})}$. Thus we have $\mathcal{Y}_{0}^{0,0, u, v}=\mathcal{Y}_{0}^{S, \tilde{u}, \tilde{v}}$, where,

$$
\begin{aligned}
& X_{t}^{u, v}=\int_{0}^{t} \sigma\left(s, \tilde{u}_{s}, \tilde{v}_{s}\right)\left[d B_{s}+b\left(s, \tilde{u}_{s}, \tilde{v}_{s}\right) d s\right] ; \\
& \mathcal{Y}_{t}^{S, \tilde{u}, \tilde{v}}=\xi\left(X^{u, v}\right)+\int_{t}^{T} f\left(s, X^{\tilde{u}, \tilde{v}}, \mathcal{Y}_{s}^{S, \tilde{u}, \tilde{v}}, \widehat{\mathcal{Z}}_{s}^{S, \tilde{u}, \tilde{v}}, \tilde{u}_{s}, \tilde{v}_{s}\right) d s-\int_{t}^{T} \mathcal{Z}_{s}^{S, \tilde{u}, \tilde{v}} d B_{s},
\end{aligned}
$$


However, we shall emphasize that the mapping from $(u, v)$ to $(\tilde{u}, \tilde{v})$ is in pairs, and it does not induce a mapping from $u$ to $\tilde{u}$ (or from $v$ to $\tilde{v}$ ). Consequently the game values defined below in strong formulation are different from the $\underline{Y}_{0}$ and $\bar{Y}_{0}$ in (3.10):

$$
\underline{Y}_{0}^{S}:=\sup _{\tilde{u} \in \mathcal{U}_{0}} \inf _{\tilde{v} \in \mathcal{V}_{0}} \mathcal{Y}_{0}^{S, \tilde{u}, \tilde{v}}, \quad \bar{Y}_{0}^{S}:=\inf _{\tilde{v} \in \mathcal{V}_{0}} \sup _{\tilde{u} \in \mathcal{U}_{0}} \mathcal{Y}_{0}^{S, \tilde{u}, \tilde{v}}
$$

Indeed, in strong formulation the above game with control against control may not have the game value, namely $\underline{Y}_{0}^{S}<\bar{Y}_{0}^{S}$, even if Isaacs condition and comparison principle for the viscosity solutions of the corresponding Bellman-Isaacs equation hold. See the counterexample Example 8.1 below.

Remark 3.8 (i) In standard literature, see e.g. [19] and [5], one transforms the problem into a game with strategy type of controls. That is, let $\alpha: \mathcal{V}_{t} \rightarrow \mathcal{U}_{t}$ and $\beta: \mathcal{U}_{t} \rightarrow \mathcal{V}_{t}$ be appropriate strategies. One considers:

$$
\underline{Y}^{\prime \prime}(t, \omega):=\operatorname{supinf}_{\alpha} \mathcal{Y}_{t}^{t, \omega, \alpha(v), v}, \quad \bar{Y}^{\prime \prime}(t, \omega):=\inf _{\beta} \sup _{u} \mathcal{Y}_{t}^{t, \omega, u, \beta(u)},
$$

This type of control problem is in fact a principal-agent problem, see e.g. Cvitanic and Zhang [11. In Markovian framework and under appropriate conditions, one can show that $\underline{Y}^{\prime \prime}=\bar{Y}$ " and is the unique solution of the corresponding Bellman-Isaacs equation. However, in this formulation the two players have nonsymmetric informations, and the lower and upper values are defined using different information settings. In particular, it is less convenient to define saddle point in this formulation.

(ii) Our weak formulation actually has the feature of strategy type of controls. Indeed, consider the $(\tilde{u}, \tilde{v})$ in Remark 3.7 again. Roughly speaking, given $u$, then $\tilde{u}$ is uniquely determined by $v$, which is in turn uniquely determined by $\tilde{v}$. Thus $u$ can be viewed as a strategy $\alpha$ which maps $\tilde{v}$ (and $B$ ) to $\tilde{u}$. Similarly $v$ can be viewed as a strategy $\beta$ which maps $\tilde{u}$ (and $B$ ) to $\tilde{v}$. Compared to the strategy against control, the advantage of weak formulation is that it is control against control and the two players have symmetric information.

Remark 3.9 When there is only drift control, namely $\sigma$ is independent of $(u, v)$, our formulation reduces to the work Hamadene and Lepeltier [21. Under Isaacs condition, by using Girsanov transformation and comparison for BSDEs, they proved $\underline{Y}=\bar{Y}$ and the existence of saddle point. We allow for both diffusion control and drift control, and we shall prove $\underline{Y}=\bar{Y}$. However, when there is diffusion control, the comparison used in [21] fails. Consequently, we are not able to follow the arguments in [21] to establish the existence of saddle point. Indeed, with the presence of diffusion control, even for stochastic optimization problem the optimal control does not seem to exist in general. We shall instead obtain some approximate saddle point in Section 7 below. 


\section{Dynamic Programming Principle}

We start with the regularity of $\underline{Y}$ and $\bar{Y}$ in $\omega$. This property is straightforward in strong formulation. Our proof here relies heavily on our assumption that $\sigma$ and $b$ are independent of $\omega$. As pointed out in Remark 3.6 (ii), the problem is very subtle in general case and we hope to address it in some future research.

Lemma 4.1 Let Assumptions 3.1, 3.3, and 3.5 hold. Then $\underline{Y}$ and $\bar{Y}$ are uniformly continuous in $\omega$ with modulus of continuity function $C \rho_{0}$ for some constant $C>0$. Consequently, $\underline{Y}$ and $\bar{Y}$ are $\mathbb{F}$-progressively measurable.

Proof. Let $t \in[0, T], \omega, \omega^{\prime} \in \Omega$. For any $(u, v) \in \mathcal{U}_{t} \times \mathcal{V}_{t}$, denote $\Delta \mathcal{Y}:=\mathcal{Y}^{t, \omega, u, v}-$ $\mathcal{Y}^{t, \omega^{\prime}, u, v}, \Delta \mathcal{Z}:=\mathcal{Z}^{t, \omega, u, v}-\mathcal{Z}^{t, \omega^{\prime}, u, v}$. Then, $\mathbb{P}^{t, u, v}$-a.s.

$$
\begin{aligned}
\Delta \mathcal{Y}_{s}= & \xi^{t, \omega}\left(B^{t}\right)-\xi^{t, \omega^{\prime}}\left(B^{t}\right)-\int_{s}^{T} \Delta \mathcal{Z}_{r} d B_{r}+\int_{s}^{T}\left[\alpha_{r} \Delta \mathcal{Y}_{r}+\Delta \widehat{\mathcal{Z}}_{r} \sigma\left(r, u_{r}, v_{r}\right) \beta_{r}\right. \\
& \left.+\left[f^{t, \omega}\left(r, B^{t}, \mathcal{Y}_{r}^{t, \omega, u, v}, \widehat{\mathcal{Z}}_{r}^{t, \omega, u, v}, u_{r}, v_{r}\right)-f^{t, \omega^{\prime}}\left(r, B^{t}, \mathcal{Y}_{r}^{t, \omega, u, v}, \widehat{\mathcal{Z}}_{r}^{t, \omega, u, v}, u_{r}, v_{r}\right)\right]\right] d r
\end{aligned}
$$

where $\alpha$ and $\beta$ are bounded. Apply (3.8) on the above BSDE, one obtains

$$
\left|\mathcal{Y}_{t}^{t, \omega, u, v}-\mathcal{Y}_{t}^{t, \omega^{\prime}, u, v}\right| \leq C \rho_{0}\left(\left\|\omega-\omega^{\prime}\right\|_{t}\right)
$$

Thus

$$
\left|\underline{Y}_{t}(\omega)-\underline{Y}_{t}\left(\omega^{\prime}\right)\right| \leq \sup _{(u, v) \in \mathcal{U}_{t} \times \mathcal{V}_{t}}\left|\mathcal{Y}_{t}^{t, \omega, u, v}-\mathcal{Y}_{t}^{t, \omega^{\prime}, u, v}\right| \leq C \rho_{0}\left(\left\|\omega-\omega^{\prime}\right\|_{t}\right) .
$$

Similarly one can prove the estimate for $\bar{Y}$.

The following Dynamical Programming Principle is important for us.

Lemma 4.2 Let Assumptions 3.1, 3.3, and 3.5 hold true. For any $0 \leq s \leq t \leq T$ and $\omega \in \Omega$ we have

$$
\underline{Y}_{s}(\omega)=\sup _{u \in \mathcal{U}_{s}} \inf _{v \in \mathcal{V}_{s}} \mathcal{Y}_{s}^{s, \omega, u, v}\left[t, \underline{Y}_{t}^{s, \omega}\right] ; \quad \bar{Y}_{s}(\omega)=\inf _{v \in \mathcal{V}_{s}} \sup _{u \in \mathcal{U}_{s}} \mathcal{Y}_{s}^{s, \omega, u, v}\left[t, \bar{Y}_{t}^{s, \omega}\right]
$$

To prove the lemma we need a technical lemma. Its proof is standard but lengthy, and is postponed to Appendix in order not to distract our main arguments.

Lemma 4.3 For any $\varepsilon>0$ and $t \in(0, T)$, there exist disjoint sets $\left\{E_{i}, i=1, \cdots, n\right\} \subseteq \mathcal{F}_{t}$ such that

$$
\left\|\omega-\omega^{\prime}\right\|_{t} \leq \varepsilon \text { for all } \omega, \omega^{\prime} \in E_{i}, i=1, \cdots, n, \quad \text { and } \sup _{(u, v) \in \mathcal{U}_{0} \times \mathcal{V}_{0}} \mathbb{E}^{\mathbb{P}^{0, u, v}}\left(\cap_{i=1}^{n} E_{i}^{c}\right) \leq \varepsilon
$$


Proof of Lemma 4.2. We shall prove only the Dynamic Programming Principle for $\underline{Y}$. The proof for $\bar{Y}$ is similar. Without loss of generality, we assume $s=0$. That is, we shall prove:

$$
\underline{Y}_{0}=\sup _{u \in \mathcal{U}_{0}} \inf _{v \in \mathcal{V}_{0}} \mathcal{Y}_{0}^{0,0, u, v}\left[t, \underline{Y}_{t}\right]
$$

Step 1. We first prove " $\geq "$. Fix arbitrary $\varepsilon>0$ and $u \in \mathcal{U}_{0}$. Let $\left\{E_{i}, i=1, \cdots, n\right\} \subset \mathcal{F}_{t}$ be given by Lemma 4.3, and fix an $\omega^{i} \in E_{i}$ for each $i$. For any $\omega \in E_{i}$, By Lemma 4.1 and (4.1) we have

$$
\left|\underline{Y}_{t}(\omega)-\underline{Y}_{t}\left(\omega^{i}\right)\right| \leq C \rho_{0}(\varepsilon) \quad \text { and } \quad \sup _{(u, v) \in \mathcal{U}_{t} \times \mathcal{V}_{t}}\left|\mathcal{Y}_{t}^{t, \omega, u, v}-\mathcal{Y}_{t}^{t, \omega^{i}, u, v}\right| \leq C \rho_{0}(\varepsilon) .
$$

Let $u^{i} \in \mathcal{U}_{t}$ be an $\varepsilon$-optimizer of $\underline{Y}_{t}\left(\omega^{i}\right)$, that is,

$$
\inf _{v \in \mathcal{V}_{t}} \mathcal{Y}_{t}^{t, \omega^{i}, u^{i}, v}+\varepsilon \geq \underline{Y}_{t}\left(\omega^{i}\right) .
$$

Denote $\hat{E}_{n}:=\cap_{i=1}^{n}\left(E_{i}\right)^{c}$. By Lemma 3.2 (i) we define $u^{\varepsilon} \in \mathcal{U}_{0}$ by:

$$
u_{s}^{\varepsilon}(\omega):=u_{s}(\omega) \mathbf{1}_{[0, t)}(s)+\left[\sum_{i=1}^{n} u_{s}^{i}\left(\omega^{t}\right) \mathbf{1}_{E_{i}}(\omega)+u_{s}(\omega) \mathbf{1}_{\hat{E}_{n}}(\omega)\right] \mathbf{1}_{[t, T]}(s)
$$

Now for any $v \in \mathcal{V}_{0}$, we have

$$
\mathcal{Y}_{0}^{0, \mathbf{0}, u^{\varepsilon}, v}=\mathcal{Y}_{0}^{0, \mathbf{0}, u^{\varepsilon}, v}\left(t, \mathcal{Y}_{t}^{0, \mathbf{0}, u^{\varepsilon}, v}\right)=\mathcal{Y}_{0}^{0, \mathbf{0}, u^{\varepsilon}, v}\left[t, \sum_{i=1}^{n} \mathcal{Y}_{t}^{0, \mathbf{0}, u^{\varepsilon}, v} \mathbf{1}_{E_{i}}+\mathcal{Y}_{t}^{0, \mathbf{0}, u^{\varepsilon}, v} \mathbf{1}_{\hat{E}^{n}}\right]
$$

Since solutions of BSDEs can be constructed via Picard iteration, one can easily check that, for any $(u, v) \in \mathcal{U}_{0} \times \mathcal{V}_{0}$,

$$
\mathcal{Y}_{t}^{0, \mathbf{0}, u, v}(\omega)=\mathcal{Y}_{t}^{t, \omega, u^{t, \omega}, v^{t, \omega}}, \quad \mathbb{P}^{0, u, v} \text {-a.e. } \omega \in \Omega .
$$

Then it follows from (4.4) and Lemma 2.2 that, for $\mathbb{P}^{0, u^{\varepsilon}, v}$-a.e. $\omega \in E_{i}$,

$$
\begin{aligned}
\mathcal{Y}_{t}^{0, \mathbf{0}, u^{\varepsilon}, v}(\omega) & =\mathcal{Y}_{t}^{t, \omega,\left(u^{\varepsilon}\right)^{t, \omega}, v^{t, \omega}}=\mathcal{Y}_{t}^{t, \omega, u^{i}, v^{t, \omega}} \geq \inf _{v \in \mathcal{V}_{t}} \mathcal{Y}_{t}^{t, \omega, u^{i}, v} \\
& \geq \inf _{v \in \mathcal{V}_{t}} \mathcal{Y}_{t}^{t, \omega^{i}, u^{i}, v}-C \rho_{0}(\varepsilon) \geq \underline{Y}_{t}\left(\omega^{i}\right)-\varepsilon-C \rho_{0}(\varepsilon) \\
& \geq \underline{Y}_{t}(\omega)-\varepsilon-C \rho_{0}(\varepsilon) .
\end{aligned}
$$

Therefore, by comparison principle of BSDEs and (3.11) we have

$$
\begin{aligned}
\mathcal{Y}_{0}^{0, \mathbf{0}, u^{\varepsilon}, v} & \geq \mathcal{Y}_{0}^{0, \mathbf{0}, u^{\varepsilon}, v}\left[t, \sum_{i=1}^{n} \underline{Y}_{t} \mathbf{1}_{E_{i}}-\left(\varepsilon+C \rho_{0}(\varepsilon)\right)+\mathcal{Y}_{t}^{0, \mathbf{0}, u^{\varepsilon}, v} \mathbf{1}_{\hat{E}^{n}}\right] \\
& \geq \mathcal{Y}_{0}^{0, \mathbf{0}, u^{\varepsilon}, v}\left[t, \underline{Y}_{t}-\left(\varepsilon+C \rho_{0}(\varepsilon)\right)-C \mathbf{1}_{\hat{E}^{n}}\right] .
\end{aligned}
$$


Recall that $\sup _{(u, v) \in \mathcal{U}_{0} \times \mathcal{V}_{0}} \mathbb{P}^{0, u, v}\left(\hat{E}_{n}\right) \leq \varepsilon$. Applying (3.9) we get

$$
\mathcal{Y}_{0}^{0, \mathbf{0}, u^{\varepsilon}, v} \geq \mathcal{Y}_{0}^{0, \mathbf{0}, u^{\varepsilon}, v}\left[t, \underline{Y}_{t}\right]-C\left(\varepsilon+\rho_{0}(\varepsilon)\right)^{\frac{1}{2}}=\mathcal{Y}_{0}^{0, \mathbf{0}, u, v}\left[t, \underline{Y}_{t}\right]-C\left(\varepsilon+\rho_{0}(\varepsilon)\right)^{\frac{1}{2}} \text {. }
$$

Since $v$ is arbitrary, this implies that

$$
\inf _{v \in \mathcal{V}_{0}} \mathcal{Y}_{0}^{0, \mathbf{0}, u^{\varepsilon}, v} \geq \inf _{v \in \mathcal{V}_{0}} \mathcal{Y}_{0}^{0, \mathbf{0}, u, v}\left[t, \underline{Y}_{t}\right]-C\left(\varepsilon+\rho_{0}(\varepsilon)\right)^{\frac{1}{2}}
$$

Then

$$
\underline{Y}_{0} \geq \inf _{v \in \mathcal{V}_{0}} \mathcal{Y}_{0}^{0, \mathbf{0}, u, v}\left[t, \underline{Y}_{t}\right]-C\left(\varepsilon+\rho_{0}(\varepsilon)\right)^{\frac{1}{2}} .
$$

Sending $\varepsilon \rightarrow 0$ and by the arbitrariness of $u \in \mathcal{U}_{0}$, we obtain

$$
\underline{Y}_{0} \geq \sup _{u \in \mathcal{U}_{0}} \inf _{v \in \mathcal{V}_{0}} \mathcal{Y}_{0}^{0,0, u, v}\left[t, \underline{Y}_{t}\right]
$$

Step 2. We now prove " $\leq$ ". Fix $\bar{u} \in \mathcal{U}_{0}$ in the form of (3.5), with $u_{i j}$ being replaced by $\bar{u}_{i j}$. It suffices to prove that

$$
\inf _{v \in \mathcal{V}_{0}} \mathcal{Y}_{0}^{t, \mathbf{0}, \bar{u}, v} \leq \inf _{v \in \mathcal{V}_{0}} \mathcal{Y}_{0}^{0,0, \bar{u}, v}\left[t, \underline{Y}_{t}\right]
$$

Without loss of generality, assume $t=t_{i_{0}}$ for some $i_{0}$. Notice that $\underline{Y}_{t_{m}}=\xi$, then it suffices to prove

$$
\inf _{v \in \mathcal{V}_{0}} \mathcal{Y}_{0}^{0, \mathbf{0}, \bar{u}, v}\left[t_{i+1}, \underline{Y}_{t_{i+1}}\right] \leq \inf _{v \in \mathcal{V}_{0}} \mathcal{Y}_{0}^{0, \mathbf{0}, \bar{u}, v}\left[t_{i}, \underline{Y}_{t_{i}}\right], \quad \text { for all } i
$$

We now fix $i$ and recall that $\bar{u}_{t}=\sum_{j=1}^{n_{i}} \bar{u}_{i j} \mathbf{1}_{E_{j}^{i}}$ for $t \in\left[t_{i}, t_{i+1}\right)$. For any $\varepsilon>0$, let $\left\{E_{k}, k=1, \cdots, K\right\} \subset \mathcal{F}_{t_{i}}$ be given by Lemma 4.3. Denote $E_{j k}^{i}:=E_{j}^{i} \cap E_{k}$ and fix an $\omega^{j k} \in E_{j k}^{i}$ for each $(j, k)$. For any $\bar{v} \in \mathcal{V}_{0}$, as in Step1 we have

$$
\begin{aligned}
\mathcal{Y}_{0}^{0, \mathbf{0}, \bar{u}, \bar{v}}\left[t_{i}, \underline{Y}_{t_{i}}\right] & =\mathcal{Y}_{0}^{0, \mathbf{0}, \bar{u}, \bar{v}}\left[t_{i}, \sum_{j, k=1}^{n_{i}, K} \underline{Y}_{t_{i}} \mathbf{1}_{E_{j k}^{i}}+\underline{Y}_{t_{i}} \mathbf{1}_{\cap_{k=1}^{K} E_{k}^{c}}\right] \\
& \geq \mathcal{Y}_{0}^{0, \mathbf{0}, \bar{u}, \bar{v}}\left[t_{i}, \sum_{j, k=1}^{n_{i}, K} \underline{Y}_{t_{i}}\left(\omega^{j k}\right) \mathbf{1}_{E_{j k}^{i}}(\omega)\right]-C\left(\rho_{0}(\varepsilon)+\varepsilon\right)^{\frac{1}{2}} .
\end{aligned}
$$

By Step 1, we see that

$$
\underline{Y}_{t_{i}}\left(\omega^{j k}\right) \geq \sup _{u \in \mathcal{U}_{t_{i}}} \inf _{v \in \mathcal{V}_{t_{i}}} \mathcal{Y}_{t_{i}}^{t_{i}, \omega^{j k}, u, v}\left[t_{i+1}, \underline{Y}_{t_{i+1}}^{t_{i}, \omega^{j k}}\right] \geq \inf _{v \in \mathcal{V}_{t_{i}}} \mathcal{Y}_{t_{i}}^{t_{i}, \omega^{j k}, \bar{u}_{i j}, v}\left[t_{i+1}, \underline{Y}_{t_{i+1}}^{t_{i}, \omega^{j k}}\right]
$$

Here the constant $\bar{u}_{i j}$ denotes the constant process. Then there exists $v^{j k} \in \mathcal{V}_{t_{i}}$ such that

$$
\underline{Y}_{t_{i}}\left(\omega^{j k}\right) \geq \mathcal{Y}_{t_{i}}^{t_{i}, \omega^{j k}, \bar{u}_{i j}, \omega^{j k}}\left[t_{i+1}, \underline{Y}_{t_{i+1}}^{t_{i}, \omega^{j k}}\right]-\varepsilon .
$$


Now define

$$
\hat{v}:=\bar{v} \mathbf{1}_{\left[0, t_{i}\right)}+\left[\sum_{j, k=1}^{n_{i}, K} v^{j k}\left(B^{t_{i}}\right) \mathbf{1}_{E_{j k}^{i}}+\bar{v} \mathbf{1}_{\cap_{k=1}^{K} E_{k}^{c}}\right] \mathbf{1}_{\left[t_{i}, T\right]} .
$$

By Lemma 3.2 we have $\hat{v} \in \mathcal{V}_{0}$. Then, noting that $\bar{u}_{t}^{t_{i}, \omega}=\bar{u}_{i j}$ for $\omega \in E_{j k}^{i}$ and $t \in\left[t_{i}, t_{i+1}\right)$,

$$
\begin{aligned}
& \mathcal{Y}_{0}^{0, \mathbf{0}, \bar{u}, \bar{v}}\left[t_{i}, \underline{Y}_{t_{i}}\right] \geq \mathcal{Y}_{0}^{0, \mathbf{0}, \bar{u}, \bar{v}}\left[\sum_{j, k=1}^{n_{i}, K} \mathcal{Y}_{t_{i}}^{t_{i}, \omega^{j k}, \bar{u}_{i j}, v^{j k}}\left[t_{i+1}, \underline{Y}_{t_{i+1}}^{t_{i}, \omega^{j k}}\right] \mathbf{1}_{E_{j k}^{i}}(\omega)\right]-C\left(\rho_{0}(\varepsilon)+\varepsilon\right)^{\frac{1}{2}} \\
& =\mathcal{Y}_{0}^{0, \mathbf{0}, \bar{u}, \bar{v}}\left[\sum_{j, k=1}^{n_{i}, K} \mathcal{Y}_{t_{i}}^{t_{i}, \omega^{j k}, \bar{u}^{t_{i}, \omega}, \hat{v}^{t_{i}, \omega}}\left[t_{i+1}, \underline{Y}_{t_{i+1}}^{t_{i}, \omega^{j k}}\right] \mathbf{1}_{E_{j k}^{i}}(\omega)\right]-C\left(\rho_{0}(\varepsilon)+\varepsilon\right)^{\frac{1}{2}} \\
& \geq \mathcal{Y}_{0}^{0, \mathbf{0}, \bar{u}, \bar{v}}\left[\sum_{j, k=1}^{n_{i}, K} \mathcal{Y}_{t_{i}}^{t_{i}, \omega, \bar{u}_{i}^{t_{i}, \omega}, \hat{v}_{i}^{t_{i}, \omega}}\left[t_{i+1}, \underline{Y}_{t_{i+1}}^{t_{i}, \omega}\right] \mathbf{1}_{E_{j k}^{i}}(\omega)\right]-C\left(\rho_{0}(\varepsilon)+\varepsilon\right)^{\frac{1}{2}} \\
& =\mathcal{Y}_{0}^{0, \mathbf{0}, \bar{u}, \hat{v}}\left[t_{i+1}, \sum_{j, k=1}^{n_{i}, K} \underline{Y}_{t_{i+1}}^{t_{i}, \omega} \mathbf{1}_{E_{j k}^{i}}(\omega)\right]-C\left(\rho_{0}(\varepsilon)+\varepsilon\right)^{\frac{1}{2}} \\
& \geq \mathcal{Y}_{0}^{0, \mathbf{0}, \bar{u}, \hat{v}}\left[t_{i+1}, \underline{Y}_{t_{i+1}}\right]-C\left(\rho_{0}(\varepsilon)+\varepsilon\right)^{\frac{1}{2}} \\
& \geq \inf _{v \in \mathcal{V}_{0}} \mathcal{Y}_{0}^{0, \mathbf{0}, \bar{u}, v}\left[t_{i+1}, \underline{Y}_{t_{i+1}}\right]-C\left(\rho_{0}(\varepsilon)+\varepsilon\right)^{\frac{1}{2}} \text {. }
\end{aligned}
$$

Send $\varepsilon \rightarrow 0$, by the arbitrariness of $\bar{v} \in \mathcal{V}_{0}$ we prove (4.6).

Remark 4.4 If we use strong formulation with control against control, as in Remark 3.7, we can only prove the following partial Dynamic Programming Principle:

$$
\underline{Y}_{s}^{S}(\omega) \leq \sup _{u \in \mathcal{U}_{s}} \inf _{v \in \mathcal{V}_{s}} \mathcal{Y}^{s, \omega, \mathbb{P}^{S, s, u, v}}\left[\underline{Y}_{t}^{S}\right] ; \quad \bar{Y}_{s}^{S}(\omega) \geq \inf _{v \in \mathcal{V}_{s}} \sup _{u \in \mathcal{U}_{s}} \mathcal{Y}^{s, \omega, \mathbb{P}^{S, s, u, v}}\left[\bar{Y}_{t}^{S}\right]
$$

which does not lead to the desired viscosity property. That is why we use weak formulation instead of strong formulation.

We now turn to the regularity of $\underline{Y}$ and $\bar{Y}$ in $t$, which is required for studying their viscosity property.

Lemma 4.5 Let Assumptions 3.1, 3.3, and 3.5 hold. Then, for any $0 \leq t_{1}<t_{2} \leq T$ and $\omega \in \Omega$,

$$
\left|\underline{Y}_{t_{1}}(\omega)-\underline{Y}_{t_{2}}(\omega)\right|+\left|\bar{Y}_{t_{1}}(\omega)-\bar{Y}_{t_{2}}(\omega)\right| \leq C \rho_{1}\left(d_{\infty}\left(\left(t_{1}, \omega\right),\left(t_{2}, \omega\right)\right)\right),
$$

where $\rho_{1}$ is a modulus of continuity function defined by

$$
\rho_{1}(\delta):=\rho_{0}\left(\delta+\delta^{\frac{1}{4}}\right)+\delta+\delta^{\frac{1}{4}} .
$$


Proof. We shall only prove the regularity of $\underline{Y}$ in $t$. The estimate for $\bar{Y}$ can be proved similarly. Denote $\delta:=d_{\infty}\left(\left(t_{1}, \omega\right),\left(t_{2}, \omega\right)\right)$.

By Theorem 4.6 and Lemma 4.1 we have

$$
\begin{aligned}
\left|\underline{Y}_{t_{1}}(\omega)-\underline{Y}_{t_{2}}(\omega)\right| & =\left|\sup _{u \in \mathcal{U}_{t_{1}}} \inf _{v \in \mathcal{V}_{t_{1}}} \mathcal{Y}_{t_{1}}^{t_{1}, \omega, u, v}\left[t_{2}, \underline{Y}_{t_{2}}^{t_{1}, \omega}\right]-\underline{Y}_{t_{2}}(\omega)\right| \\
& \leq \sup _{u \in \mathcal{U}_{t_{1}}, v \in \mathcal{V}_{t_{1}}}\left|\mathcal{Y}_{t_{1}}^{t_{1}, \omega, u, v}\left[t_{2}, \underline{Y}_{t_{2}}^{t_{1}, \omega}\right]-\underline{Y}_{t_{2}}(\omega)\right|
\end{aligned}
$$

Denote

$$
\mathcal{Y}_{t}:=\mathcal{Y}_{t}^{t_{1}, \omega, u, v}\left[t_{2}, \underline{Y}_{t_{2}}^{t_{1}, \omega}\right]-\underline{Y}_{t_{2}}(\omega), \quad \mathcal{Z}_{t}:=\mathcal{Z}_{t}^{t_{1}, \omega, u, v}\left[t_{2}, \underline{Y}_{t_{2}}^{t_{1}, \omega}\right]
$$

Then, $\mathbb{P}^{t_{1}, u, v}$-a.s.

$$
\mathcal{Y}_{t}=\underline{Y}_{t_{2}}^{t_{1}, \omega}-\underline{Y}_{t_{2}}(\omega)+\int_{t}^{t_{2}} f^{t_{1}, \omega}\left(s, B^{t_{1}}, \mathcal{Y}_{s}+\underline{Y}_{t_{2}}(\omega), \widehat{\mathcal{Z}}_{s}, u_{s}, v_{s}\right) d s-\int_{t}^{t_{2}} \mathcal{Z}_{s} d B_{s}^{t_{1}} .
$$

Recall from (3.11) that $\underline{Y}$ is bounded. Apply (3.9) and Lemma 4.1, we get

$$
\begin{aligned}
\left|\mathcal{Y}_{t_{1}}\right| & \leq C\left(\mathbb{E}^{\mathbb{P}^{t_{1}, u, v}}\left[\left|\underline{Y}_{t_{2}}^{t_{1}, \omega}-\underline{Y}_{t_{2}}(\omega)\right|^{2}\right]\right)^{\frac{1}{2}}+C \delta \\
& \leq C\left(\mathbb{E}^{\mathbb{P}^{t_{1}, u, v}}\left[\rho_{0}^{2}\left(d_{\infty}\left(\left(t_{2}, \omega\right),\left(t_{2}, \omega \otimes_{t_{1}} B^{t_{1}}\right)\right)\right)\right]\right)^{\frac{1}{2}}+C \delta .
\end{aligned}
$$

Note that

$$
\begin{aligned}
& \left.\mathbb{E}^{\mathbb{P}^{t_{1}, u, v}}\left[\rho_{0}^{2}\left(d_{\infty}\left(\left(t_{2}, \omega\right),\left(t_{2}, \omega \otimes_{t_{1}} B^{t_{1}}\right)\right)\right)\right] \leq \mathbb{E}^{\mathbb{P}^{t_{1}, u, v}}\left[\rho_{0}^{2}\left(\delta+\left\|B^{t_{1}}\right\|_{t_{2}}\right)\right]\right] \\
\leq & \rho_{0}^{2}\left(\delta+\delta^{\frac{1}{4}}\right)+C \mathbb{P}^{t_{1}, u, v}\left[\left\|B^{t_{1}}\right\|_{t_{2}} \geq \delta^{\frac{1}{4}}\right] \leq \rho_{0}^{2}\left(\delta+\delta^{\frac{1}{4}}\right)+C \delta^{-\frac{1}{2}} \mathbb{E}^{\mathbb{P}^{t_{1}, u, v}}\left[\left\|B^{t_{1}}\right\|_{t_{2}}^{2}\right] \\
\leq & \rho_{0}^{2}\left(\delta+\delta^{\frac{1}{4}}\right)+C \delta^{\frac{1}{2}} .
\end{aligned}
$$

Then

$$
\left|\mathcal{Y}_{t_{1}}\right| \leq C\left[\rho_{0}\left(\delta+\delta^{\frac{1}{4}}\right)+\delta^{\frac{1}{4}}+\delta\right]=C \rho_{1}(\delta)
$$

Plug this into (4.9) we complete the proof.

Combining Lemmas 4.2 and 4.5, it follows from standard arguments that

Theorem 4.6 Let Assumptions 3.1, 3.3, and 3.5 hold true. For any $(t, \omega) \in \Lambda$ and $\tau \in \mathcal{T}^{t}$, we have

$$
\underline{Y}_{t}(\omega)=\sup _{u \in \mathcal{U}_{t}} \inf _{v \in \mathcal{V}_{t}} \mathcal{Y}_{s}^{t, \omega, u, v}\left[\tau, \underline{Y}_{\tau}^{t, \omega}\right] ; \quad \bar{Y}_{t}(\omega)=\inf _{v \in \mathcal{V}_{t}} \sup _{u \in \mathcal{U}_{t}} \mathcal{Y}_{s}^{t, \omega, u, v}\left[\tau, \bar{Y}_{\tau}^{t, \omega}\right]
$$




\section{$5 \quad$ Viscosity solution properties}

Define

$$
\begin{aligned}
& \underline{G}(t, \omega, y, z, \gamma):=\sup _{u \in \mathbb{U}} \inf _{v \in \mathbb{V}}\left[\frac{1}{2} \sigma^{2}(t, u, v): \gamma+b \sigma(t, u, v) z+f(t, \omega, y, z \sigma(t, u, v), u, v)\right] \\
& \bar{G}(t, \omega, y, z, \gamma):=\inf _{v \in \mathbb{V}} \sup _{u \in \mathbb{U}}\left[\frac{1}{2} \sigma^{2}(t, u, v): \gamma+b \sigma(t, u, v) z+f(t, \omega, y, z \sigma(t, u, v), u, v)\right]
\end{aligned}
$$

and consider the following path dependent PDEs:

$$
\begin{array}{r}
-\partial_{t} Y_{t}-\underline{G}\left(t, \omega, Y_{t}, \partial_{\omega} Y_{t}, \partial_{\omega \omega}^{2} Y_{t}\right)=0 \\
-\partial_{t} Y_{t}-\bar{G}\left(t, \omega, Y_{t}, \partial_{\omega} Y_{t}, \partial_{\omega \omega}^{2} Y_{t}\right)=0 .
\end{array}
$$

Theorem 5.1 Let Assumptions [3.1, 3.3, and 3.5 hold. Then $\underline{Y}$ (resp. $\bar{Y}$ ) is a viscosity solution of PPDE (5.2) (resp. (5.3)).

Proof. We shall only prove that $\underline{Y}$ is a viscosity solution of the PPDE (5.2). The other statement can be proved similarly.

Step 1. We first prove the viscosity supersolution property. Assume by contradiction that there exists $(t, \omega)$ and $\varphi \in \overline{\mathcal{A}}^{L} \underline{Y}(t, \omega)$ such that

$$
\begin{aligned}
c:= & \partial_{t} \varphi(t, \mathbf{0})+\sup _{u \in \mathbb{U}} \inf _{v \in \mathbb{V}}\left\{\frac{1}{2} \sigma^{2}(t, u, v): \partial_{\omega \omega}^{2} \varphi(t, \mathbf{0})+b \sigma(t, u, v) \partial_{\omega} \varphi(t, \mathbf{0})\right. \\
& \left.+f\left(t, \omega, \underline{Y}_{t}(\omega), \partial_{\omega} \varphi(t, \mathbf{0}) \sigma(t, u, v), u, v\right)\right\}>0 .
\end{aligned}
$$

By Remark (2.5), we can assume $L$ is large enough as we will see later. Then there exists $\tilde{u} \in \mathbb{U}$ such that, for all $v \in \mathbb{V}$

$$
\begin{aligned}
& \partial_{t} \varphi(t, \mathbf{0})+\frac{1}{2} \sigma^{2}(t, \tilde{u}, v): \partial_{\omega \omega}^{2} \varphi(t, \mathbf{0})+b \sigma(t, \tilde{u}, v) \partial_{\omega} \varphi(t, \mathbf{0}) \\
& +f\left(t, \omega, \underline{Y}_{t}(\omega), \partial_{\omega} \varphi(t, \mathbf{0}) \sigma(t, \tilde{u}, v), \tilde{u}, v\right) \geq \frac{c}{2}
\end{aligned}
$$

Let $\mathrm{H} \in \mathcal{H}^{t}$ be the hitting time corresponding to $\varphi$ in (3.2). For any $\varepsilon>0$, set

$$
\mathrm{H}_{\varepsilon}:=\inf \left\{s \geq t: s-t+\left|B_{s}^{t}\right|=\varepsilon\right\} .
$$

By choosing $\varepsilon>0$ small enough, we have $\mathrm{H}_{\varepsilon} \leq \mathrm{H}$. Since $\varphi \in C^{1,2}\left(\Lambda^{t}\right)$, there exist some constant $C_{\varphi} \geq C_{0}$ and modulus of continuity function $\rho_{\varphi} \geq \rho_{1}$, which may depend on $\varphi$, such that

$$
\left|\psi\left(s, B^{t}\right)\right| \leq C_{\varphi},\left|\psi\left(s, B^{t}\right)-\psi(t, \mathbf{0})\right| \leq \rho_{\varphi}(\varepsilon) \text {, for } t \leq s \leq \mathrm{H}_{\varepsilon}, \psi=\varphi, \partial_{t} \varphi, \partial_{\omega} \varphi, \partial_{\omega \omega}^{2} \varphi .
$$

Now set $u:=\tilde{u} \in \mathcal{U}_{t}$ be a constant process and let $v \in \mathcal{V}_{t}$ be arbitrary. Fix $\delta>0$ and denote $\mathrm{H}_{\varepsilon}^{\delta}:=\mathrm{H}_{\varepsilon} \wedge(t+\delta)$,

$$
\begin{aligned}
& \mathcal{Y}:=\mathcal{Y}^{t, \omega, u, v}\left[\mathrm{H}_{\varepsilon}^{\delta}, \underline{Y}_{\mathrm{H}_{\varepsilon}^{\delta}}^{t, \omega}\right], \quad \mathcal{Z}:=\mathcal{Z}^{t, \omega, u, v}\left[\mathrm{H}_{\varepsilon}^{\delta}, \underline{Y}_{\mathrm{H}_{\varepsilon}^{\delta}}^{t, \omega}\right] \\
& \Delta Y_{s}:=\varphi\left(s, B^{t}\right)-\mathcal{Y}_{s}, \quad \Delta Z_{s}:=\partial_{\omega} \varphi\left(s, B^{t}\right)-\mathcal{Z}_{s} .
\end{aligned}
$$


Then, applying the functional Itô's formula we obtain:

$$
\begin{aligned}
d \Delta Y_{s}= & {\left[\partial_{t} \varphi+\frac{1}{2} \partial_{\omega \omega}^{2} \varphi: \sigma^{2}\left(s, u_{s}, v_{s}\right)+f^{t, \omega}\left(\cdot, \mathcal{Y}_{s}, \widehat{\mathcal{Z}}_{s}, u_{s}, v_{s}\right)\right]\left(s, B^{t}\right) d s+\Delta Z_{s} d B_{s}^{t} } \\
= & {\left[\partial_{t} \varphi+\frac{1}{2} \partial_{\omega \omega}^{2} \varphi: \sigma^{2}\left(s, u_{s}, v_{s}\right)+f^{t, \omega}\left(\cdot, \mathcal{Y}_{s}, \widehat{\mathcal{Z}}_{s}, u_{s}, v_{s}\right)\right]\left(s, B^{t}\right) d s+\Delta Z_{s} d B_{s}^{t} } \\
= & {\left[\partial_{t} \varphi+\frac{1}{2} \partial_{\omega \omega}^{2} \varphi: \sigma^{2}\left(s, u_{s}, v_{s}\right)+f^{t, \omega}\left(\cdot, \underline{Y}_{t}(\omega), \partial_{\omega} \varphi(\cdot) \sigma\left(s, u_{s}, v_{s}\right), u_{s}, v_{s}\right)\right]\left(s, B^{t}\right) d s } \\
& +\left[\alpha_{s}\left(\mathcal{Y}_{s}-\underline{Y}_{t}(\omega)\right)+\Delta \widehat{Z}_{s} \beta_{s}\right] d s+\Delta Z_{s} d B_{s}^{t},
\end{aligned}
$$

where $|\alpha|,|\beta| \leq L_{0}$. By (5.4) and (5.5) we have

$$
d \Delta Y_{s} \geq\left[\frac{c}{2}-C_{\varphi} \rho_{\varphi}(\varepsilon)-C\left|\mathcal{Y}_{s}-\underline{Y}_{t}(\omega)\right|+\Delta \widehat{Z}_{s} \beta_{s}\right] d s+\Delta Z_{s} d B_{s}^{t}, \quad t \leq s \leq \mathrm{H}_{\varepsilon}^{\delta} .
$$

Recall (3.4) and define $d \overline{\mathbb{P}}:=M_{\mathrm{H}_{\varepsilon}^{\delta}} d \mathbb{P}^{t, u, v}$, where

$$
M_{s}:=\exp \left(\int_{t}^{s}\left[b\left(r, u_{r}, v_{r}\right)+\beta_{r}\right] d W_{r}^{t, u, v}-\frac{1}{2} \int_{t}^{s}\left|b\left(r, u_{r}, v_{r}\right)+\beta_{r}\right|^{2} d r\right) .
$$

Then $\Delta Z_{s} d B_{s}^{t}+\Delta \widehat{Z}_{s} \beta_{s} d s$ is a $\overline{\mathbb{P}}$-martingale, and thus

$$
\Delta Y_{t} \leq \mathbb{E}^{\overline{\mathbb{P}}}\left[\Delta Y_{\mathrm{H}_{\varepsilon}^{\delta}}-\int_{t}^{\mathrm{H}_{\varepsilon}^{\delta}}\left[\frac{c}{2}-C_{\varphi} \rho_{\varphi}(\varepsilon)-C\left|\mathcal{Y}_{s}-\underline{Y}_{t}(\omega)\right|\right] d s\right]
$$

By choosing $L$ large enough, we see that $\overline{\mathbb{P}} \in \mathcal{P}_{L}^{t}$. Then it follows from the definition of $\overline{\mathcal{A}}^{L} \underline{Y}(t, \omega)$ that

$$
\mathbb{E}^{\overline{\mathbb{P}}}\left[\Delta Y_{\mathrm{H}_{\varepsilon}^{\delta}}\right]=\mathbb{E}^{\overline{\mathbb{P}}}\left[\varphi\left(\mathrm{H}_{\varepsilon}^{\delta}, B^{t}\right)-\underline{Y}_{\mathrm{H}_{\varepsilon}^{\delta}}^{t, \omega}\right] \leq \varphi(t, \mathbf{0})-\underline{Y}_{t}(\omega) .
$$

Therefore, since $b$ and $\beta$ are bounded,

$$
\begin{aligned}
& \underline{Y}_{t}(\omega)-\mathcal{Y}_{t} \leq \mathbb{E}^{\overline{\mathbb{P}}}\left[\int_{t}^{\mathrm{H}_{\varepsilon}^{\delta}}\left[-\frac{c}{2}+C_{\varphi} \rho_{\varphi}(\varepsilon)+C\left|\mathcal{Y}_{s}-\underline{Y}_{t}(\omega)\right|\right] d s\right] \\
\leq & {\left[-\frac{c}{2}+C_{\varphi} \rho_{\varphi}(\varepsilon)\right] \delta+C_{\varphi} \delta \overline{\mathbb{P}}\left(\mathrm{H}_{\varepsilon} \leq t+\delta\right)+C \delta \mathbb{E}^{\overline{\mathbb{P}}}\left[\left\|\mathcal{Y} .-\underline{Y}_{t}(\omega)\right\|_{\mathrm{H}_{\varepsilon}^{\delta}}\right] } \\
\leq & {\left[-\frac{c}{2}+C_{\varphi} \rho(\varepsilon)\right] \delta+C_{\varphi} \delta\left(\mathbb{P}^{t, u, v}\left(\mathrm{H}_{\varepsilon} \leq t+\delta\right)\right)^{\frac{1}{2}}+C \delta\left(\mathbb{E}^{\mathbb{P}^{t, u, v}}\left[\left\|\mathcal{Y} .-\underline{Y}_{t}(\omega)\right\|_{\mathrm{H}_{\varepsilon}^{\delta}}^{2}\right]\right)^{\frac{1}{2}}(.5 .6}
\end{aligned}
$$

Note that, for $\delta \leq \frac{\varepsilon}{2}$,

$$
\begin{aligned}
\mathbb{P}^{t, u, v}\left(\mathrm{H}_{\varepsilon} \leq t+\delta\right) & \leq \mathbb{P}^{t, u, v}\left(\delta+\left\|B^{t}\right\|_{t+\delta} \geq \varepsilon\right)=\mathbb{P}^{t, u, v}\left(\left\|B^{t}\right\|_{t+\delta} \geq \frac{\varepsilon}{2}\right) \\
& \leq \frac{C}{\varepsilon^{2}} \mathbb{E}^{\mathbb{P}^{t, u, v}}\left[\left\|B^{t}\right\|_{t+\delta}^{2}\right] \leq \frac{C \delta}{\varepsilon^{2}}
\end{aligned}
$$

Moreover, denote $\tilde{\mathcal{Y}}:=\mathcal{Y}-\underline{Y}_{t}(\omega)$. Then

$$
\tilde{\mathcal{Y}}_{s}=\underline{Y}_{\mathrm{H}_{\varepsilon}^{\delta}}^{t, \omega}-\underline{Y}_{t}(\omega)+\int_{s}^{\mathrm{H}_{\varepsilon}^{\delta}} f^{t, \omega}\left(r, B^{r}, \tilde{\mathcal{Y}}_{r}+\underline{Y}_{t}(\omega), \widehat{\mathcal{Z}}_{r}, u_{r}, v_{r}\right) d r-\int_{s}^{\mathrm{H}_{\varepsilon}^{\delta}} \mathcal{Z}_{r} d B_{r}^{t} .
$$


By (3.9) and applying Lemma 4.5 we obtain

$$
\begin{aligned}
& \mathbb{E}^{\mathbb{P}^{t, u, v}}\left[\left\|\mathcal{Y} .-\underline{Y}_{t}(\omega)\right\|_{\mathrm{H}_{\varepsilon}^{\delta}}^{2}\right] \leq C \mathbb{E}^{\mathbb{P}^{t, u, v}}\left[\left|\underline{Y}_{\mathrm{H}_{\varepsilon}^{\delta}}^{t, \omega}-\underline{Y}_{t}(\omega)\right|^{2}\right]+C \delta \\
\leq & C \delta+C \mathbb{E}^{\mathbb{P}^{t, u, v}}\left[\rho_{1}^{2}\left(d_{\infty}\left((t, \omega),\left(t+\delta, \omega \otimes_{t} B^{t}\right)\right)\right)\right] \\
\leq & \left.C \delta+C \mathbb{E}^{\mathbb{P}^{t, u, v}}\left[\rho_{1}^{2}\left(d_{\infty}((t, \omega),(t+\delta, \omega))+\left\|B^{t}\right\|_{t+\delta}\right)\right)\right] \leq C \rho_{2}(\delta),
\end{aligned}
$$

where

$$
\left.\rho_{2}(\delta):=\delta+\sup _{(u, v) \in \mathcal{U}_{t} \times \mathcal{V}_{t}} \mathbb{E}^{\mathbb{P}^{t, u, v}}\left[\rho_{1}^{2}\left(d_{\infty}((t, \omega),(t+\delta, \omega))+\left\|B^{t}\right\|_{t+\delta}\right)\right)\right] .
$$

Plug (5.7) and (5.8) into (5.6), we have

$$
\underline{Y}_{t}(\omega)-\mathcal{Y}_{t} \leq \delta\left[-\frac{c}{2}+C_{\varphi} \rho_{\varphi}(\varepsilon)+\frac{C_{\varphi} \delta^{\frac{1}{2}}}{\varepsilon}+C \delta \rho_{2}^{\frac{1}{2}}(\delta)\right] .
$$

It is clear that $\lim _{\delta \rightarrow 0} \rho_{2}(\delta)=0$. Then by first choosing $\varepsilon$ small and then choosing $\delta$ small enough, we have

$$
\underline{Y}_{t}(\omega)-\mathcal{Y}_{t}^{t, \omega, u, v}\left[\mathrm{H}_{\varepsilon}^{\delta}, \underline{Y}_{\mathrm{H}_{\varepsilon}^{\delta}}^{t, \omega}\right] \leq-\frac{c}{4} \delta .
$$

Since $v$ is arbitrary, we get

$$
\underline{Y}_{t}(\omega)-\inf _{v \in \mathcal{V}_{t}} \mathcal{Y}_{t}^{t, \omega, u, v}\left[\mathrm{H}_{\varepsilon}^{\delta}, \underline{Y}_{\mathrm{H}_{\varepsilon}^{\delta}}^{t, \omega}\right] \leq-\frac{c}{4} \delta
$$

which implies further that

$$
\underline{Y}_{t}(\omega)-\sup _{u \in \mathcal{U}_{t}} \inf _{v \in \mathcal{V}_{t}} \mathcal{Y}_{t}^{t, \omega, u, v}\left[\mathrm{H}_{\varepsilon}^{\delta}, \underline{Y}_{\mathrm{H}_{\varepsilon}^{\delta}}^{t, \omega}\right] \leq-\frac{c}{4} \delta<0 .
$$

This contradicts with the dynamic programming principle Theorem 4.6. Therefore, $\underline{Y}$ is a viscosity supersolution of PPDE (5.2).

Step 2. We now prove the viscosity subsolution property. Assume by contradiction that, for some $L$ large enough, there exists $(t, \omega)$ and $\varphi \in \underline{\mathcal{A}}^{L} \underline{Y}(t, \omega)$ such that

$$
\begin{aligned}
-c:= & \partial_{t} \varphi(t, \mathbf{0})+\sup _{u \in \mathbb{U}} \inf _{v \in \mathbb{V}}\left\{\frac{1}{2} \sigma^{2}(t, u, v): \partial_{\omega \omega}^{2} \varphi(t, \mathbf{0})+b(t, u, v) \partial_{\omega} \varphi(t, \mathbf{0})\right\} \\
& \left.+f\left(t, \omega, \underline{Y}_{t}(\omega), \partial_{\omega} \varphi(t, \mathbf{0}), u, v\right)\right\}<0 .
\end{aligned}
$$

Then there exists a mapping (no measurability is involved!) $\psi: \mathbb{U} \rightarrow \mathbb{V}$ such that, for any $u \in \mathbb{U}$,

$$
\begin{aligned}
& \partial_{t} \varphi(t, \mathbf{0})+\frac{1}{2} \sigma^{2}(t, u, \psi(u)): \partial_{\omega \omega}^{2} \varphi(t, \mathbf{0})+b(t, u, \psi(u)) \partial_{\omega} \varphi(t, \mathbf{0}) \\
& +f\left(t, \omega, \underline{Y}_{t}(\omega), \partial_{\omega} \varphi(t, \mathbf{0}), u, \psi(u)\right) \leq-\frac{c}{2} .
\end{aligned}
$$


For any $u \in \mathcal{U}_{t}$, by the structure (3.5) one can easily see that $v:=\psi(u) \in \mathcal{V}_{t}$. Introduce the same notations as in Step 1, and follow almost the same arguments, we obtain

$$
\underline{Y}_{t}(\omega)-\mathcal{Y}_{t} \geq \delta\left[\frac{c}{2}-C_{\varphi} \rho_{\varphi}(\varepsilon)-\frac{C_{\varphi} \delta^{\frac{1}{2}}}{\varepsilon}-C \delta \rho_{2}^{\frac{1}{2}}(\delta)\right] .
$$

Again, by first choosing $\varepsilon$ small and then choosing $\delta$ small enough, we have

$$
\underline{Y}_{t}(\omega)-\mathcal{Y}_{t}^{t, \omega, u, v}\left[\mathrm{H}_{\varepsilon}^{\delta}, \underline{Y}_{H_{\varepsilon}^{\delta}}^{t, \omega}\right] \geq \frac{c}{4} \delta .
$$

This implies

$$
\underline{Y}_{t}(\omega)-\inf _{v \in \mathcal{V}_{t}} \mathcal{Y}_{t}^{t, \omega, u, v}\left[\mathrm{H}_{\varepsilon}^{\delta}, \underline{Y}_{\mathrm{H}_{\varepsilon}^{\delta}}^{t, \omega}\right] \geq \frac{c}{4} \delta .
$$

Since $u$ is arbitrary, then

$$
\underline{Y}_{t}(\omega)-\sup _{u \in \mathcal{U}_{t}} \inf _{v \in \mathcal{V}_{t}} \mathcal{Y}_{t}^{t, \omega, u, v}\left[\mathrm{H}_{\varepsilon}^{\delta}, \underline{Y}_{\mathrm{H}_{\varepsilon}^{\delta}}^{t, \omega}\right] \geq \frac{c}{4} \delta>0 .
$$

This contradicts with the dynamic programming principle Theorem 4.6. Therefore, $\underline{Y}$ is a viscosity subsolution of PPDE (5.2).

We now assume the Isaacs condition:

$$
\underline{G}(t, \omega, y, z, \gamma)=\bar{G}(t, \omega, y, z, \gamma)=: G(t, \omega, y, z, \gamma),
$$

and consider the following path dependent Isaacs equation:

$$
-\partial_{t} Y_{t}-G\left(t, \omega, Y_{t}, \partial_{\omega} Y_{t}, \partial_{\omega \omega}^{2} Y_{t}\right)=0
$$

Our main result of the paper is:

Theorem 5.2 Let Assumptions [3.1, 3.3, and 3.5 hold. Assume further that the Isaacs condition (5.11) and the uniqueness for viscosity solutions of the PPDE (5.12) hold. Then $\underline{Y}=\bar{Y}=: Y$ and is the unique viscosity solution of PPDE (5.12).

Proof. Applying Theorem 5.1 and by the uniqueness of viscosity solutions, we see immediately that $\underline{Y}=\bar{Y}$ and it is the unique viscosity solution of PPDE (5.12).

Remark 5.3 (i) For the comparison principle of viscosity solutions of PPDE (5.12), we refer to Ekren, Touzi and Zhang [15]. We shall also provide a sufficient condition in Subsection 6 below.

(ii) In Markovian framework, the PPDE (5.12) becomes a standard PDE. Note that a viscosity solution (resp. supersolution, subsolution) in the sense of Definition 2.4 is a viscosity solution (resp. supersolution, subsolution) in the standard literature. Then, assuming the comparison principle for standard viscosity solution of PDEs holds true, $Y:=\underline{Y}=\bar{Y}$ and is the unique viscosity solution of the Bellman-Isaacs PDE with terminal condition $Y(T, x)=\xi(x)$ 


\section{Comparison principle for viscosity solutions of PPDEs}

In this section we study the comparison principle of PPDE (5.12), which clearly implies the uniqueness required in Theorem 5.1,

We first cite a general result from [17] concerning wellposedness of PPDEs, adapting to our setting. For any $(t, \omega) \in \Lambda$, denote the following deterministic function with parameter $(t, \omega)$ :

$$
g^{t, \omega}(s, y, z, \gamma):=G(s \wedge T, \omega \cdot \wedge t, y, z, \gamma)
$$

For any $\varepsilon>0$ and $\eta \geq 0$, we denote $T_{\eta}:=(1+\eta) T$, and

$$
\begin{aligned}
& O_{\varepsilon}:=\left\{x \in \mathbb{R}^{d}:|x|<\varepsilon\right\}, \quad \bar{O}_{\varepsilon}:=\left\{x \in \mathbb{R}^{d}:|x| \leq \varepsilon\right\}, \quad \partial O_{\varepsilon}:=\left\{x \in \mathbb{R}^{d}:|x|=\varepsilon\right\} ; \\
& \left.\mathcal{O}_{t}^{\varepsilon, \eta}:=\left[t, T_{\eta}\right) \times O_{\varepsilon}, \overline{\mathcal{O}}_{t}^{\varepsilon, \eta}:=\left[t, T_{\eta}\right] \times \bar{O}_{\varepsilon}\right), \partial \mathcal{O}_{t}^{\varepsilon, \eta}:=\left(\left[t, T_{\eta}\right] \times \partial O_{\varepsilon}\right) \cup\left(\left\{T_{\eta}\right\} \times O_{\varepsilon}\right),
\end{aligned}
$$

Consider the following localized and path-frozen PDE defined for every $(t, \omega) \in \Lambda$ :

$$
(\mathrm{E})_{\varepsilon, \eta}^{t, \omega} \quad \mathbf{L}^{t, \omega} t h:=-\partial_{t} \theta-g^{t, \omega}\left(s, \theta, D \theta, D^{2} \theta\right)=0 \text { on } \mathcal{O}_{t}^{\varepsilon, \eta} .
$$

Here $\partial_{t}, D, D^{2}$ are standard differential operators.

Assumption 6.1 For any $\varepsilon>0, \eta \geq 0,(t, \omega) \in \Lambda$, and any $h \in C^{0}\left(\partial \mathcal{O}_{t}^{\varepsilon, \eta}\right)$, we have $\bar{\theta}=\underline{\theta}$, where

$$
\begin{aligned}
& \bar{\theta}(s, x):=\inf \left\{w(s, x): w \text { classical supersolution of }(\mathrm{E})_{\varepsilon, \eta}^{t, \omega} \text { and } w \geq h \text { on } \partial \mathcal{O}_{t}^{\varepsilon, \eta}\right\}, \\
& \underline{\theta}(s, x):=\sup \left\{w(s, x): w \text { classical subsolution of }(\mathrm{E})_{\varepsilon, \eta}^{t, \omega} \text { and } w \leq h \text { on } \partial \mathcal{O}_{t}^{\varepsilon, \eta}\right\} .
\end{aligned}
$$

By [17] Theorem 3.4, we have

Theorem 6.2 Let Assumptions 3.1, 3.3, 3.5, and the Isaacs condition (5.11) hold. Then, under the additional Assumption 6.1, the PPDE (5.12) has a unique viscosity solution and the comparison principle of viscosity solutions holds.

We remark that Assumption 6.1 is in the spirit of Perron's approach. However, in standard literature the $w$ in (6.4) is required only to be viscosity supersolution or subsolution, while we require it to be a classical one. To check that, we present a result concerning classical solutions of parabolic PDEs.

We first simplify the notations. Let $O \subset \mathbb{R}^{d}$ be open, connected, bounded, and with smooth boundary. Set

$$
\mathcal{O}:=[0, T) \times O, \quad \overline{\mathcal{O}}:=[0, T] \times \bar{O}, \quad \partial \mathcal{O}:=([0, T] \times \partial O) \cup(\{T\} \times O) .
$$


Consider the following (standard) PDE in $\mathcal{O}$ with boundary condition $h$ :

$$
-\partial_{t} \theta-g\left(t, x, \theta, D \theta, D^{2} \theta\right)=0 \text { in } \mathcal{O} \text { and } \theta=h \text { on } \partial \mathcal{O} \text {. }
$$

Then we have the following result, whose argument is standard in the literature and is communicated to us by Lihe Wang. We present its proof in Appendix for completeness.

Lemma 6.3 Assume

(i) $h \in C^{1,2}(\overline{\mathcal{O}})$ and $g(\cdot, y, z, \gamma) \in C^{1,2}(\overline{\mathcal{O}})$ for any $(y, z, \gamma)$;

(ii) $g$ is continuously differentiable in $(y, z, \gamma)$ with bounded derivatives;

(iii) $\partial_{\gamma} g \geq c_{0} I_{d}$ for some $c_{0}>0$, and $d \leq 2$.

Then the PDE (6.5) has a classical solution $\theta \in C^{1,2}(\overline{\mathcal{O}})$.

We now have

Proposition 6.4 Let Assumptions 3.1, 3.3, 3.5, and the Isaacs condition (5.11) hold. Assume further that

$$
\sigma \geq c_{0} I_{d} \text { for some } c_{0}>0 \text { and the dimension } d \leq 2 \text {. }
$$

Then Assumption 6.1 holds true. Consequently $\underline{Y}=\bar{Y}=: Y$ and is the unique viscosity solution of PPDE (5.12).

Proof. We use the notations in Assumption 6.1. By [16] Proposition 3.14, we may assume without loss of generality that

$$
G\left(\cdot, y_{1}, \cdot\right)-G\left(\cdot, y_{2}, \cdot\right) \leq y_{2}-y_{1} \quad \text { for any } \quad y_{1} \geq y_{2}
$$

First, one can easily extend $h$ to a uniformly continuous function on $[t, \infty) \times \mathbb{R}^{d}$, still denoted as $h$. For any $\delta>0$, let $g_{\delta}^{t, \omega}$ and $h_{\delta}$ be smooth mollifiers of $g^{t, \omega}$ and $h$ such that $\left\|g_{\delta}^{t, \omega}-g\right\|_{\infty} \leq \delta,\left\|h_{\delta}-h\right\|_{\infty} \leq \delta$. By our assumptions, it is clear that $c_{0} I_{d} \leq \partial_{\gamma} g_{\delta}^{t, \omega} \leq L_{0} I_{d}$. Apply Lemma 6.3, the following PDE has a classical solution $\theta_{\delta} \in C^{1,2}\left(\overline{\mathcal{O}}_{t}^{\varepsilon, \eta}\right)$ :

$$
-\partial_{t} \theta_{\delta}-g_{\delta}^{t, \omega}\left(s, \theta_{\delta}, D \theta_{\delta}, D^{2} \theta_{\delta}\right)=0, \quad \text { in } \mathcal{O}_{t}^{\varepsilon, \eta}, \quad \theta_{\delta}=h_{\delta} \quad \text { on } \partial \mathcal{O}_{t}^{\varepsilon, \eta} .
$$

Denote

$$
\bar{\theta}_{\delta}:=\theta_{\delta}+\delta \quad \text { and } \quad \underline{\theta}_{\delta}:=\theta_{\delta}-\delta .
$$

Then clearly $\bar{\theta}_{\delta} \in C^{1,2}\left(\overline{\mathcal{O}}_{t}^{\varepsilon, \eta}\right), \bar{\theta}_{\delta} \geq h$ on $\partial \mathcal{O}_{t}^{\varepsilon, \eta}$. Moreover, by (6.7)

$$
\begin{aligned}
\mathbf{L}^{t, \omega} \bar{\theta}_{\delta} & =-\partial_{t} \theta_{\delta}-g^{t, \omega}\left(s, \theta_{\delta}+\delta, D \theta_{\delta}, D^{2} \theta_{\delta}\right) \\
& \geq-\partial_{t} \theta_{\delta}-g^{t, \omega}\left(s, \theta_{\delta}, D \theta_{\delta}, D^{2} \theta_{\delta}\right)+\delta \\
& =g_{\delta}^{t, \omega}\left(s, \theta_{\delta}, D \theta_{\delta}, D^{2} \theta_{\delta}\right)-g^{t, \omega}\left(s, \theta_{\delta}, D \theta_{\delta}, D^{2} \theta_{\delta}\right)+\delta \geq 0 .
\end{aligned}
$$


Then $\bar{\theta}_{\delta}$ is a classical supersolution of $(\mathrm{E})_{\varepsilon, \eta}^{t, \omega}$, and thus $\bar{\theta} \leq \bar{\theta}_{\delta}$. Similarly, $\underline{\theta} \leq \underline{\theta}_{\delta}$. Then

$$
0 \leq \bar{\theta}-\underline{\theta} \leq \bar{\theta}_{\delta}-\underline{\theta}_{\delta}=2 \delta
$$

Since $\delta>0$ is arbitrary, we conclude that $\bar{\theta}=\underline{\theta}$.

\section{Approximate saddle point}

In this section we discuss briefly saddle points of the game, assuming the game value exists. In our setting, it is natural to define

Definition 7.1 We call $\left(u^{*}, v^{*}\right) \in \mathcal{U}_{0} \times \mathcal{V}_{0}$ a saddle point of the game if

$$
\mathcal{Y}_{0}^{0,0, u, v^{*}} \leq \mathcal{Y}_{0}^{0, \mathbf{0}, u^{*}, v^{*}} \leq \mathcal{Y}_{0}^{0,0, u^{*}, v} \quad \text { for all } \quad u \in \mathcal{U}_{0}, v \in \mathcal{V}_{0}
$$

We remark that, if a saddle point $\left(u^{*}, v^{*}\right)$ exists, then it is straightforward to check that the game has a value $Y_{0}:=\mathcal{Y}_{0}^{0, \mathbf{0}, u^{*}, v^{*}}$. However, even in stochastic optimization problem with diffusion control, in general the optimal control may not exist. We thus study approximate saddle points only.

Definition 7.2 For any $\varepsilon>0$, we call $\left(u^{\varepsilon}, v^{\varepsilon}\right) \in \mathcal{U}_{0} \times \mathcal{V}_{0}$ an $\varepsilon$-saddle point of the game if

$$
\mathcal{Y}_{0}^{0, \mathbf{0}, u, v^{\varepsilon}}-\varepsilon \leq \mathcal{Y}_{0}^{0, \mathbf{0}, u^{\varepsilon}, v^{\varepsilon}} \leq \mathcal{Y}_{0}^{0,0, u^{\varepsilon}, v}+\varepsilon \quad \text { for all } \quad u \in \mathcal{U}_{0}, v \in \mathcal{V}_{0}
$$

We have the following simple observation:

Proposition 7.3 Assume the game has a value, then it has an $\varepsilon$-saddle point $\left(u^{\varepsilon}, v^{\varepsilon}\right)$ for any $\varepsilon>0$.

Proof. Let $\underline{Y}_{0}=Y_{0}=\bar{Y}_{0}$ be the game value. Then for any $\varepsilon>0$, there exist $u^{\varepsilon} \in \mathcal{U}_{0}, v^{\varepsilon} \in$ $\mathcal{V}_{0}$ such that

$$
Y_{0}-\varepsilon<\inf _{v \in \mathcal{V}_{0}} \mathcal{Y}_{0}^{0, \mathbf{0}, u^{\varepsilon}, v} \leq Y_{0} \leq \sup _{u \in \mathcal{U}_{0}} \mathcal{Y}_{0}^{0, \mathbf{0}, u, v^{\varepsilon}} \leq Y_{0}+\varepsilon
$$

In particular, this implies that

$$
Y_{0}-\varepsilon<\inf _{v \in \mathcal{V}_{0}} \mathcal{Y}_{0}^{0, \mathbf{0}, u^{\varepsilon}, v} \leq \mathcal{Y}_{0}^{0,0, u^{\varepsilon}, v^{\varepsilon}} \leq \sup _{u \in \mathcal{U}_{0}} \mathcal{Y}_{0}^{0,0, u, v^{\varepsilon}} \leq Y_{0}+\varepsilon
$$

That is, $\left(u^{\varepsilon}, v^{\varepsilon}\right)$ is an $\varepsilon$-saddle point. Moreover, we observe that $\left|\mathcal{Y}_{0}^{0,0, u^{\varepsilon}, v^{\varepsilon}}-Y_{0}\right| \leq \varepsilon$. 


\section{Appendix}

\subsection{Proof of Lemma 2.2}

We prove the case $\overline{\mathcal{A}}^{S}$ only. The case $\overline{\mathcal{A}}^{W}$ can be proved similarly. Let $X$ be the unique strong solution to $\mathrm{SDE}(2.7)$ with coefficients $(\sigma, b)$, and $X^{i}$ be the unique strong solution to SDE (2.7) on $[t, T]$ with coefficients $\left(\sigma^{i}, b^{i}\right)$.

First, denote

$$
\bar{X}_{s}=X_{s} \mathbf{1}_{[0, t)}(s)+\left[X_{t}+\sum_{i=1}^{n} \mathbf{1}_{E_{i}}(X) X_{s}^{i}\left(B^{t}\right)\right] \mathbf{1}_{[t, T]}(s), \quad 0 \leq s \leq T .
$$

One can check straightforwardly that $\bar{X}$ is a strong solution to SDE (2.7) with coefficients $(\bar{\sigma}, \bar{b})$. On the other hand, let $\tilde{X}$ be an arbitrary strong solution to SDE (2.7) with coefficients $(\bar{\sigma}, \bar{b})$. Then both $\bar{X}$ and $\tilde{X}$ satisfy $\operatorname{SDE}(2.7)$ on $[0, t]$ with coefficients $(\sigma, b)$. By the uniqueness assumption of $(\sigma, b)$, we see that $\bar{X}=\tilde{X}$ on $[0, t], \mathbb{P}_{0}$-a.s. In particular, this implies $\mathbf{1}_{E_{i}}(\bar{X})=\mathbf{1}_{E_{i}}(\tilde{X})$. Then for $\mathbb{P}_{0}$-a.e. $\omega \in \Omega$, there exists unique $i$ such that $\mathbf{1}_{E_{i}}(\bar{X})=$ $\mathbf{1}_{E_{i}}(\tilde{X})=1$. Thus both $\bar{X}^{t, \omega}$ and $\tilde{X}^{t, \omega}$ satisfy SDE (2.7) on $[t, T]$ with coefficients $\left(\sigma^{i}, b^{i}\right)$.

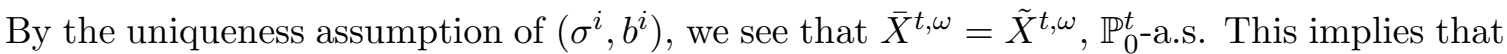
$\bar{X}=\tilde{X}, \mathbb{P}_{0}$-a.s. and therefore, $(\bar{\sigma}, \bar{b}) \in \overline{\mathcal{A}}^{S}$.

Finally, since $\bar{X}=X$ on $[0, t]$, we have $\mathbb{P}^{\bar{\sigma}, \bar{b}}=\mathbb{P}^{\sigma, b}$ on $\mathcal{F}_{t}$. Moreover, since $\bar{X}^{t, \omega}\left(B^{t}\right)=$ $X_{t}(\omega)+X^{i}\left(B^{t}\right)$ whenever $\mathbf{1}_{E_{i}}(X)=1$, by the definition of r.c.p.d. we see that $\left(\mathbb{P}^{\bar{\sigma}, \bar{b}}\right)^{t, \omega}=$ $\mathbb{P}^{t, \sigma^{i}, b^{i}}$ for $\mathbb{P}^{\sigma, b}$-a.e. $\omega \in E_{i}$.

\subsection{Proof of Lemma 3.4}

Recall the $\mathbb{P}^{t, u, v}$-Brownian motion $W^{t, u, v}$ defined in (3.4). One may rewrite BSDE (3.7) as

$$
\mathcal{Y}_{s}=\eta+\int_{s}^{\tau}\left[f^{t, \omega}\left(r, B^{t}, \mathcal{Y}_{r}, \widehat{\mathcal{Z}}_{r}, u_{r}, v_{r}\right)+\widehat{\mathcal{Z}}_{r} b\left(r, u_{r}, v_{r}\right)\right] d r-\int_{s}^{\tau} \widehat{\mathcal{Z}}_{r} d W_{r}^{t, u, v}, \mathbb{P}^{t, u, v} \text {-a.s. }
$$

Then (3.8) follows from standard BSDE arguments. Moreover, note that

$$
\mathcal{Y}_{s}=\eta+\int_{s}^{\tau}\left[f^{t, \omega}\left(r, B^{t}, 0, \mathbf{0}, u_{r}, v_{r}\right)+\alpha_{r} \mathcal{Y}_{r}+\widehat{\mathcal{Z}}_{r} \beta_{r}\right] d r-\int_{s}^{\tau} \widehat{\mathcal{Z}}_{r} d W_{r}^{t, u, v}, \mathbb{P}^{t, u, v} \text {-a.s. }
$$

where $\alpha, \beta$ are bounded. Denote

$$
\Gamma_{r}:=\exp \left(\int_{t}^{r} \beta_{s} d W_{s}^{t, u, v}+\int_{t}^{r}\left[\alpha_{r}-\frac{1}{2}\left|\beta_{r}\right|^{2}\right] d r\right) .
$$

Then

$$
\mathcal{Y}_{t}=\Gamma_{\tau} \eta+\int_{s}^{\tau} \Gamma_{r} f^{t, \omega}\left(r, B^{t}, 0, \mathbf{0}, u_{r}, v_{r}\right) d r-\int_{s}^{\tau}[\cdots] d W_{r}^{t, u, v}, \mathbb{P}^{t, u, v} \text {-a.s. }
$$


Thus

$$
\begin{aligned}
\left|\mathcal{Y}_{t}\right|= & \left|\mathbb{E}^{\mathbb{P}^{t, u, v}}\left[\Gamma_{\tau} \eta+\int_{s}^{\tau} \Gamma_{r} f^{t, \omega}\left(r, B_{.}^{t}, 0, \mathbf{0}, u_{r}, v_{r}\right) d r\right]\right| \\
\leq & \left(\mathbb{E}^{\mathbb{P}^{t, u, v}}\left[\Gamma_{\tau}^{2}\right]\right)^{\frac{1}{2}}\left(\mathbb{E}^{\mathbb{P}^{t, u, v}}\left[|\eta|^{2}\right]\right)^{\frac{1}{2}} \\
& +\delta\left(\mathbb{E}^{\mathbb{P}^{t, u, v}}\left[\|\Gamma\|_{\tau}^{2}\right]\right)^{\frac{1}{2}}\left(\mathbb{E}^{\mathbb{P}^{t, u, v}}\left[\int_{s}^{\tau}\left|f^{t, \omega}\left(r, B^{t}, 0, \mathbf{0}, u_{r}, v_{r}\right)\right|^{2} d r\right]\right)^{\frac{1}{2}} .
\end{aligned}
$$

It is clear that $\mathbb{E}^{\mathbb{P}^{t, u, v}}\left[\|\Gamma\|_{\tau}^{2}\right] \leq C$. Then (3.9) follows immediately.

\subsection{Proof of Lemma 4.3}

We introduce the following capacity $\mathcal{C}$ :

$$
\mathcal{C}(A):=\sup _{(u, v) \in \mathcal{U}_{0} \times \mathcal{V}_{0}} \mathbb{P}^{0, u, v}(A), \quad \text { for all } \quad A \in \mathcal{F}_{T}
$$

In this proof we abuse a notation a little bit by denoting $B_{r}^{s}:=B_{r}-B_{s}$ for $0 \leq s \leq r \leq t$.

Step 1. We first show that, for any $c, \delta>0$, and $R>0$,

$$
\mathcal{C}\left(\|B\|_{t}>R\right) \leq \frac{C}{R^{4}} \quad \text { and } \quad \mathcal{C}\left(\sup _{0 \leq s \leq t}\left\|B^{s}\right\|_{(s+\delta) \wedge t} \geq c\right) \leq \frac{C \delta}{c^{4}}
$$

Indeed, for any $(u, v) \in \mathcal{U}_{0} \times \mathcal{V}_{0}$ and any $0 \leq t_{1}<t_{2}$, since $\sigma$ and $b$ are bounded, then by (3.4) and applying the Burkholder-Davis-Gundy Inequality we get

$$
\begin{aligned}
\mathbb{E}^{\mathbb{P}^{0, u, v}}\left[\left\|B^{t_{1}}\right\|_{t_{2}}^{4}\right] & \left.=\left.\mathbb{E}^{\mathbb{P}^{0, u, v}}\left[\sup _{t_{1} \leq s \leq t_{2}} \mid \int_{t_{1}}^{t_{2}} \sigma\left(r, u_{r}, v_{r}\right) b\left(r, u_{r}, v_{r}\right) d r+\int_{t_{1}}^{t_{2}} \sigma\left(r, u_{r}, v_{r}\right) d W^{u, v}\right)_{r}\right|^{4}\right] \\
& \leq C \mathbb{E}^{\mathbb{P}^{0, u, v}}\left[\left(\int_{t_{1}}^{t_{2}}\left|\sigma\left(r, u_{r}, v_{r}\right) b\left(r, u_{r}, v_{r}\right)\right| d r\right)^{4}+\left(\int_{t_{1}}^{t_{2}}\left|\sigma\left(r, u_{r}, v_{r}\right)\right|^{2} d_{r}\right)^{2}\right] \\
& \leq C\left(t_{2}-t_{1}\right)^{2} .
\end{aligned}
$$

Then

$$
\mathbb{P}^{0, u, v}\left(\|B\|_{t}>R\right) \leq \frac{1}{R^{4}} \mathbb{E}^{\mathbb{P}^{0, u, v}}\left[\|B\|_{t}^{4}\right] \leq \frac{C}{R^{4}} .
$$

By the definiton of $\mathcal{C}$, this implies the first estimate in (8.2).

Next, let $0=t_{1}<\cdots<t_{m}=t$ such that $\delta \leq \Delta t_{i}<2 \delta$ for all $i$. Then

$$
\begin{aligned}
\sup _{0 \leq s \leq t}\left\|B^{s}\right\|_{(s+\delta) \wedge t} & =\max _{0 \leq i \leq m-1} \sup _{t_{i} \leq s \leq t_{i+1}} \sup _{s \leq r \leq(s+\delta) \wedge t}\left|B_{r}-B_{s}\right| \\
& \leq \max _{0 \leq i \leq m-1} \sup _{t_{i} \leq s \leq t_{i+1}} \sup _{s \leq r \leq(s+\delta) \wedge t}\left[\left|B_{r}-B_{t_{i}}\right|+\left|B_{s}-B_{t_{i}}\right|\right] \\
& \leq 2 \max _{0 \leq i \leq m-1}\left\|B^{t_{i}}\right\|_{t_{i}+3 \delta .}
\end{aligned}
$$


Then, noting that $m \leq \frac{T}{\delta}$, by (8.3) we have

$$
\begin{aligned}
\mathbb{P}^{0, u, v}\left(\sup _{0 \leq s \leq t}\left\|B^{s}\right\|_{(s+\delta) \wedge t} \geq c\right) & \leq \frac{1}{c^{4}} \mathbb{E}^{\mathbb{P}^{0, u, v}}\left[\sup _{0 \leq s \leq t}\left\|B^{s}\right\|_{(s+\delta) \wedge t}^{4}\right] \\
& \leq \frac{C}{c^{4}} \sum_{i=0}^{m-1} \mathbb{E}^{\mathbb{P}^{0, u, v}}\left[\|\left. B^{t_{i}}\right|_{t_{i}+3 \delta} ^{4}\right] \leq \frac{C}{c^{4}} m \delta^{2} \leq \frac{C \delta}{c^{4}} .
\end{aligned}
$$

By the definition of $\mathcal{C}$ we obtain the second estimate in (8.2).

Step 2. We now fix $\varepsilon>0$. For the constant $C$ in (8.2), set

$$
c:=\frac{\varepsilon}{3}, \quad \delta:=\frac{c^{4} \varepsilon}{2 C} \wedge t=\frac{\varepsilon^{5}}{162 C} \wedge t, \quad R:=\left(\frac{2 C}{\varepsilon}\right)^{\frac{1}{4}} .
$$

Let $0=t_{0}<\cdots<t_{m}=t$ such that $\delta \leq \Delta t_{i} \leq 2 \delta, i=1, \cdots, m$. Clearly there exists a partition $\left\{\tilde{E}_{j}, 1 \leq j \leq n\right\} \subset \mathcal{F}_{t}$ such that

$$
\cup_{j=1}^{n} \tilde{E}_{j}=\left\{\max _{0 \leq i \leq m}\left|B_{t_{i}}\right| \leq R+c\right\} \text { and } \max _{0 \leq i \leq m}\left|\omega_{t_{i}}-\omega_{t_{i}}^{\prime}\right| \leq \frac{\varepsilon}{3} \text { for all } \omega, \omega^{\prime} \in \tilde{E}_{i} .
$$

Now set

$$
E_{j}:=\tilde{E}_{j} \cap A, \quad \text { where } \quad A:=\left\{\sup _{0 \leq s \leq t}\left\|B^{s}\right\|_{(s+\delta) \wedge t} \leq c\right\} \in \mathcal{F}_{t} .
$$

Then for any $\omega, \omega^{\prime} \in E_{j}$,

$$
\begin{aligned}
\left\|\omega-\omega^{\prime}\right\|_{t} & =\max _{0 \leq i \leq m-1} \sup _{t_{i} \leq s \leq t_{i+1}}\left|\omega_{s}-\omega_{s}^{\prime}\right| \\
& \leq \max _{0 \leq i \leq m-1} \sup _{t_{i} \leq s \leq t_{i+1}}\left[\left|\omega_{s}-\omega_{t_{i}}\right|+\left|\omega_{s}^{\prime}-\omega_{t_{i}}^{\prime}\right|+\left|\omega_{t_{i}}-\omega_{t_{i}}^{\prime}\right|\right] \\
& \leq \max _{0 \leq i \leq m-1} \sup _{t_{i} \leq s \leq t_{i+1}}\left[\frac{\varepsilon}{3}+\frac{\varepsilon}{3}+\frac{\varepsilon}{3}\right]=\varepsilon .
\end{aligned}
$$

On the other hand,

$$
\begin{aligned}
\cap_{j=1}^{n} E_{j}^{c} & =\left(\cup_{j=1}^{n} \tilde{E}_{j}\right)^{c} \cup A^{c}=\left\{\max _{0 \leq i \leq m}\left|B_{t_{i}}\right|>R+c\right\} \cup A^{c} \\
& \subset\left(\left\{\max _{0 \leq i \leq m}\left|B_{t_{i}}\right|>R+c\right\} \cap A\right) \cup A^{c} .
\end{aligned}
$$

For each $\omega \in\left\{\max _{0 \leq i \leq m}\left|B_{t_{i}}\right|>R+c\right\} \cap A$, we have $\|\omega\|_{t}=\max _{0 \leq i \leq m-1} \sup _{t_{i} \leq s \leq t_{i+1}}\left|\omega_{s}\right| \geq \max _{0 \leq i \leq m-1} \sup _{t_{i} \leq s \leq t_{i+1}}\left[\left|\omega_{t_{i}}\right|-\left|\omega_{s}-\omega_{t_{i}}\right|\right]>(R+c)-c=R$.

That is,

$$
\left\{\max _{0 \leq i \leq m}\left|B_{t_{i}}\right|>R+c\right\} \cap A \subset\left\{\|B\|_{t}>R\right\}
$$

and therefore,

$$
\cap_{j=1}^{n} E_{j}^{c} \subset\left\{\|B\|_{t}>R\right\} \cup A^{c} .
$$

Now it follows from $(8.2)$ that $\mathcal{C}\left(\cap_{j=1}^{n} E_{j}^{c}\right) \leq \varepsilon$. 


\subsection{Proof of Lemma 6.3}

As standard in PDE literature, it suffices to provide a priori estimates. That is, we assume $\theta \in C^{1,2}(\overline{\mathcal{O}})$ satisfies PDE (6.5) , and we shall provide estimates which depends only on the parameters in our assumptions.

(i) We first establish the estimates in the case $g=g(\gamma)$. We proceed in several steps.

Step 1. We first cite a result from Ladyzenskaya et al [25]. Assume $\theta$ satisfying the following linear PDE:

$$
-\partial_{t} \theta-\frac{1}{2} A(t, x): D^{2} \theta=0
$$

where $A=\left[a_{i j}\right]_{1 \leq i, j \leq d}$ is required only to be measurable and $\mathbf{0}<c_{0} I_{d} \leq A \leq C_{0} I_{d}$. Then $\theta \in C_{\mathrm{loc}}^{\frac{\alpha}{2}, \alpha}$, where $\alpha$ depends only on $c_{0}$ and $C_{0}$.

Step 2. We next cite a result by Caffarelli [6].

The elliptic PDE $g\left(D^{2} \theta\right)=f(x)$ with $f \in C^{\alpha}$ has $C^{2, \alpha}$-solution

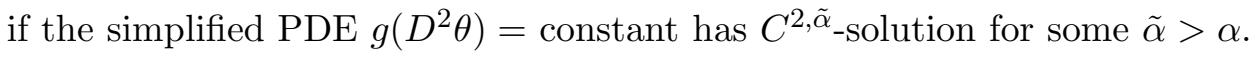

Step 3. We also need the DeGiorgi-Nash estimate: If $\sum_{i, j=1}^{d} D_{x_{i}}\left(a_{i j} D_{x_{j}} \theta\right)=0$, then $\theta \in C^{\alpha}$. See, e.g., Gilbarg and Trudinger [20] Theorem 8.22.

Step 4. We now come back to the PDE (6.5) with $g=g(\gamma)$. First, set $\tilde{\theta}:=\partial_{t} \theta$. Differentiate both sides of (6.5) with respect to $t$ we obtain:

$$
\partial_{t} \tilde{\theta}+\left[\partial_{\gamma} g\left(D^{2} \theta\right)\right]: D^{2} \tilde{\theta}=0 .
$$

By Step 1, we have $\partial_{t} \theta=\tilde{\theta} \in C^{\frac{\alpha}{2}, \alpha}$. Now fix $t$. Then (6.5) becomes

$$
g\left(D^{2} \theta\right)=-\partial_{t} \theta \in C^{\alpha}
$$

By Step 2, it suffices to show that

$$
g\left(D^{2} \theta\right)=\text { constant has } C^{2, \alpha^{\prime}} \text {-solution for some } \alpha^{\prime}>\alpha
$$

For this, we can only prove in the cases $d=1$ or $d=2$.

In the case $d=1$, notice that $g$ is strictly increasing, then $D^{2} \theta=$ constant and thus $\theta$ is a parabola.

In the case $d=2$, fix $k=1,2$ and denote $\theta^{k}:=D_{x_{k}} \theta$. Differentiate both sides of (8.4) with respect to $x_{k}$ :

$$
A: D^{2} \theta^{k}=0, \quad \text { where } A:=\left[a_{i, j}\right]_{1 \leq i, j \leq 2}:=\partial_{\gamma} g\left(D^{2} \theta\right)
$$


Note that $a_{11} \geq c_{0}>0$. Then

$$
D_{x_{1} x_{1}}^{2} \theta^{k}+\frac{a_{12}}{a_{11}} D_{x_{1} x_{2}}^{2} \theta^{k}+\frac{a_{22}}{a_{11}} D_{x_{2} x_{2}}^{2} \theta^{k}=0 .
$$

For $l=1,2$, differentiate both sides of the above PDE with respect to $x_{l}$ and denote $\theta^{k, l}:=D_{x_{l}} \theta^{k}=D_{x_{k} x_{l}}^{2} \theta$ :

$$
D_{x_{1} x_{1}}^{2} \theta^{k, l}+D_{x_{l}}\left(\frac{a_{12}}{a_{11}} D_{x_{1} x_{2}}^{2} \theta^{k}\right)+D_{x_{l}}\left(\frac{a_{22}}{a_{11}} D_{x_{2} x_{2}}^{2} \theta^{k}\right)=0 .
$$

In the case $l=2$, this is:

$$
D_{x_{1}}\left(D_{x_{1}} \theta^{k, 2}\right)+D_{x_{2}}\left(\frac{a_{12}}{a_{11}} D_{x_{1}} \theta^{k, 2}\right)+D_{x_{2}}\left(\frac{a_{22}}{a_{11}} D_{x_{2}} \theta^{k, 2}\right)=0 .
$$

By Step $3, \theta^{k, 2} \in C^{\alpha}$. Similarly, $\theta^{k, 1} \in C^{\alpha}$. That is, for any $t, \theta(t, \cdot) \in C^{2+\alpha}$. Moreover, it follows from PDE (6.5) that $\theta$ is differentiable in $t$ and thus $\theta \in C^{1,2}$.

(ii). We now consider the general case where $g=g(t, x, y, z, \gamma)$. We define a map $J: C^{1,2}(\overline{\mathcal{O}}) \rightarrow C^{1,2}(\overline{\mathcal{O}})$ by $J \theta:=\tilde{\theta}$, where, thanks to (i), $\tilde{\theta}$ is the classical solution of the following PDE:

$$
-\partial_{t} \tilde{\theta}-g\left(t, x, \theta, D \theta, D^{2} \tilde{\theta}\right)=0 \text { in } \mathcal{O} \text { and } \tilde{\theta}=\theta \text { on } \partial \mathcal{O}
$$

Now, following the arguments in [26] Theorem 8.2, one can show that the mapping $J$ is a contraction mapping if $T$ is small enough. Moreover, the fixed point $\theta$ of the mapping $J$ is also in $C^{1,2}(\overline{\mathcal{O}})$. Therefore, we can conclude the so called small time existence: the PDE (6.5) has a classical solution when $T$ is small enough.

Next, 26] Theorem 14.4 gives an a priori uniform estimate for the Hölder- $(1+\delta)$ norm of the classical solution to (6.5), for some $\delta \in(0,1)$, where the definition of the Hölder- $(1+\delta)$ norm is given in 26] Chapter IV, Section 1. Using this a priori estimate and following the arguments in [26] Theorem 8.3, we can infer the existence of the classical solution over arbitrary time duration $[0, T]$ from the small time existence, and thus complete the proof.

\subsection{Buckdahn's counterexample}

As pointed out in Remark 3.7, a game with control against control in strong formulation may not have the game value, even if the Isaacs condition and the comparison principle for the associate Bellman-Isaacs equation hold. The following counterexample is communicated to us by Rainer Buckdahn.

Example 8.1 Let $d=2, \mathbb{U}:=\{x \in \mathbb{R}:|x| \leq 1\}, \mathbb{V}:=\{x \in \mathbb{R}:|x| \leq 2\}$, and $\mathcal{U}$ (resp. $\mathcal{V}$ ) be the set of $\mathbb{F}$-progressively measurable $\mathbb{U}$-valued (resp. $\mathbb{V}$-valued) processes. Write 
$B=\left(B^{1}, B^{2}\right)$. Given $(u, v) \in \mathcal{U} \times \mathcal{V}$, the controlled state process $X^{u, v}=\left(X^{1, u}, X^{2, v}\right)$ is determined by:

$$
X_{t}^{1, u}:=\alpha B_{t}^{1}+\int_{0}^{t} u_{s} d s, \quad X_{t}^{2, v}:=\alpha B_{t}^{2}+\int_{0}^{t} v_{s} d s
$$

where $\alpha \geq 0$ is a constant. Define, for some $a \in \mathbb{R}$,

$$
J(u, v):=\mathbb{E}^{\mathbb{P}_{0}}\left[\left|a+X_{T}^{1, u}-X_{T}^{2, v}\right|\right], \quad \underline{Y}_{0}:=\sup _{u \in \mathcal{U}} \inf _{v \in \mathcal{V}} J(u, v), \quad \bar{Y}_{0}:=\inf _{v \in \mathcal{V}} \sup _{u \in \mathcal{U}} J(u, v) .
$$

Then, for $0 \leq \alpha<\sqrt{\frac{T}{2}}$ and $|a| \leq T$, we have $\underline{Y}_{0}<\bar{Y}_{0}$.

Proof. For any $u \in \mathcal{U}$, set $v_{t}:=u_{t}+\frac{a}{T}$. Then $v \in \mathcal{V}$ and,

$$
a+X_{T}^{1, u}-X_{T}^{2, v}=a+\alpha B_{T}^{1}+\int_{0}^{T} u_{t} d t-\alpha B_{T}^{2}-\int_{0}^{T}\left[u_{t}+\frac{a}{T}\right] d t=\alpha\left[B_{T}^{1}-B_{T}^{2}\right] .
$$

Thus

$$
J(u, v)=\alpha \mathbb{E}^{\mathbb{P}_{0}}\left[\left|B_{T}^{1}-B_{T}^{2}\right|\right]=\alpha \sqrt{2 T}
$$

This implies that $\inf _{v \in \mathcal{V}} J(u, v) \leq \alpha \sqrt{2 T}$. Since $u$ is arbitrary, we get

$$
\underline{Y}_{0} \leq \alpha \sqrt{2 T}
$$

On the other hand, for any $v \in \mathcal{V}$, set

$$
u_{t}:=u_{0}:=\frac{a-\mathbb{E}^{\mathbb{P}^{0}}\left[X_{T}^{2, v}\right]}{\left|a-\mathbb{E}^{\mathbb{P}^{0}}\left[X_{T}^{2, v}\right]\right|} \mathbf{1}_{\left\{a-\mathbb{E}^{\mathbb{P} 0}\left[X_{T}^{2, v}\right] \neq 0\right\}}+\mathbf{1}_{\left\{a-\mathbb{E}^{\mathbb{P} 0}\left[X_{T}^{2, v}\right]=0\right\}} .
$$

That is, $u$ is a constant process. One can easily check that

$$
u \in \mathcal{U}, \quad\left|u_{0}\right|=1, \quad a-\mathbb{E}^{\mathbb{P}^{0}}\left[X_{T}^{2, v}\right]=u_{0}\left|a-\mathbb{E}^{\mathbb{P}^{0}}\left[X_{T}^{2, v}\right]\right|
$$

Then

$$
\mathbb{E}^{\mathbb{P}_{0}}\left[a+X_{T}^{1, u}-X_{T}^{2, v}\right]=a+u_{0} T-\mathbb{E}^{\mathbb{P}_{0}}\left[X_{T}^{2, v}\right]=u_{0}\left[T+\left|a-\mathbb{E}^{\mathbb{P}^{0}}\left[X_{T}^{2, v}\right]\right|\right]
$$

Thus,

$$
\begin{aligned}
J(u, v) & \geq\left|\mathbb{E}^{\mathbb{P}_{0}}\left[a+X_{T}^{1, u}-X_{T}^{2, v}\right]\right|=\left|u_{0}\right|\left[T+\left|a-\mathbb{E}^{\mathbb{P}^{0}}\left[X_{T}^{2, v}\right]\right|\right] \\
& =T+\left|a-\mathbb{E}^{\mathbb{P}^{0}}\left[X_{T}^{2, v}\right]\right| \geq T .
\end{aligned}
$$

This $\operatorname{implies} \sup _{u \in \mathcal{U}} J(u, v) \geq T$. Since $v$ is arbitrary, we have $\bar{Y}_{0} \geq T$. This, together with 8.5. implies that $\underline{Y}_{0}<\bar{Y}_{0}$ when $0 \leq \alpha<\sqrt{\frac{T}{2}}$. 
Moreover, note that in this case the system is Markovian and $\partial_{\omega} Y=D Y$. The Hamiltonians in (5.1) become: for $(t, x, y, z, \gamma) \in[0, T] \times \mathbb{R}^{2} \times \mathbb{R} \times \mathbb{R}^{2} \times \mathbb{S}^{2}$,

$$
\begin{aligned}
\underline{G}(t, x, y, z, \gamma) & :=\sup _{u \in \mathbb{U}} \inf _{v \in \mathbb{V}}\left[\frac{1}{2} \alpha \operatorname{tr}(\gamma)+u z_{1}+v z_{2}\right]=\frac{1}{2} \alpha \operatorname{tr}(\gamma)+z_{1}^{+}-2 z_{2}^{-} \\
\bar{G}(t, x, y, z, \gamma) & :=\inf _{v \in \mathbb{V}} \sup _{u \in \mathbb{U}}\left[\frac{1}{2} \alpha \operatorname{tr}(\gamma)+u z_{1}+v z_{2}\right]=\frac{1}{2} \alpha \operatorname{tr}(\gamma)+z_{1}^{+}-2 z_{2}^{-} .
\end{aligned}
$$

Then the Isaacs condition holds, and the corresponding Bellman-Isaacs equation becomes:

$$
-\partial_{t} Y_{t}-\frac{1}{2} \alpha\left[D_{x_{1} x_{1}}^{2} Y_{t}+D_{x_{2} x_{2}}^{2} Y_{t}\right]-\left[D_{x_{1}} Y_{t}\right]^{+}+2\left[D_{x_{2}} Y_{t}\right]^{-}=0 .
$$

It is clear that the comparison principle for the viscosity solutions of above PDE holds.

Remark 8.2 (i) The above counterexample stays valid when $\alpha=0$, and thus the game is deterministic. We note that, even in deterministic case, our weak formulation is different from strong formulation. Indeed, the corresponding state process $X^{W, u, v}$ in weak formulation is:

$$
X_{t}^{W, 1, u, v}=\int_{0}^{t} u\left(s, X^{W, 1, u, v}, X^{W, 2, u, v}\right) d s, \quad X_{t}^{W, 2, u, v}=\int_{0}^{t} v\left(s, X^{W, 1, u, v}, X^{W, 2, u, v}\right) d s .
$$

In particular, $X^{W, 2, u, v}$ depends on $u$ as well. Consequently, given $v$, one cannot define $u$ through (8.6).

(ii) In this paper the drift coefficient is $b \sigma$, see (3.3), so the above deterministic example is not covered in our current framework. However, this assumption is mainly to ensure the wellposedness of the BSDE (3.7) . When $f=0$, one may define the value processes via conditional expectations, instead of $\mathcal{Y}$. Then we may consider $X$ in the form of (1.2) and all our results, after appropriate modifications, will still hold true. In particular, the above deterministic game in weak formulation has a value.

\section{References}

[1] E. Bayraktar and S. Yao. (2011) On zero-sum stochastic differential games. Preprint arXiv:1112.5744v3.

[2] Bensoussan, A., Lions, J. L. (1982) Applications of Variational Inequalities in Stochastic Control, North-Holland Publishing Company.

[3] Buckdahn, R.; Cardaliaguet, P. and Quincampoix, M. (2011) Some recent aspects of differential game theory. Dyn. Games Appl. 1, no. 1, 74-114. 
[4] Buckdahn, R.; Hu, Y. and Li, J. (2011) Stochastic representation for solutions of Isaacs' type integral-partial differential equations. Stochastic Process. Appl. 121, no. 12, 27152750.

[5] Buckdahn, R., Li, J. (2008) Stochastic Differential Games and Viscosity Solutions of Hamilton-Jacobi-Bellman-Isaacs Equations, Siam J. Control Optim., Vol.47, No.1, 444475.

[6] Caffarelli, A.L. (1989) Interior a priori estimates for solutions of fully non-linear equations, Annals of Mathematics, Vol. 130, 189-213.

[7] Cardaliaguet, P. and Rainer, C. (2009) Stochastic differential games with asymmetric information. Appl. Math. Optim. 59, no. 1, 1-36.

[8] Cheridito, P., Soner, H.M. and Touzi, N., Victoir, N. (2007) Second order BSDE's and fully nonlinear PDE's, Communications in Pure and Applied Mathematics, 60 (7): 1081-1110.

[9] Cont, R. and Fournie, D. (2012) Functional Itô calculus and stochastic integral representation of martingales, Annals of Probability, to appear, arXiv:1002.2446.

[10] Crandall, M.G, Ishii, H. and Lions, P.L. (1992) User's guide to viscosity solutions of second order partial differential equations, Bulletin of the American Mathematical Society, Vol. 27, No.1, pp. 1-67.

[11] Cvitanic, J. and Zhang, J. (2012) Contract Theory in Continuous Time Models, Springer Finance, to appear.

[12] Dupire, B. (2009) Functional Itô calculus, papers.ssrn.com.

[13] El-Karoui, N. and Hamadene, S. (2003) BSDEs and risk-sensitive control, zero-sum and nonzero-sum game problems of stochastic functional differential equations. Stochastic Process. Appl. 107, no. 1, 145-169.

[14] Ekren, I., Keller, C., Touzi, N., and Zhang, J. On Viscosity Solutions of Path Dependent PDEs, Annals of Probability, to appear, arXiv:1109.5971.

[15] Ekren, I., Touzi, N., and Zhang, J. Optimal Stopping under Nonlinear Expectation, preprint.

[16] Ekren, I., Touzi, N., and Zhang, J. Viscosity Solutions of Fully Nonlinear Parabolic Path Dependent PDEs: Part I, preprint. 
[17] Ekren, I., Touzi, N., and Zhang, J. Viscosity Solutions of Fully Nonlinear Path Parabolic Dependent PDEs: Part II, preprint.

[18] Evans, L.C. and Souganidis, P.E. (1984) Differential games and representation formulas for solutions of Hamilton-Jacobi-Isaacs equations. Indiana Univ. Math. J. 33, 773-797.

[19] Fleming, W.H., Souganidis P.E. (1989) On The Existence of Value Functions of TwoPlayer, Zero-Sum Stochastic Differential Games, Indiana University Mathematics Journal Vol. 38, No.2, 293-314.

[20] Gilbarg D. and Trudinger N.S., Elliptic Partial Differential Equations of Second Order, 2nd ed., Springer-Verlag, New York, 1983.

[21] Hamadene, S. and Lepeltier, J.P. (1995) Zero-sum stochastic differential games and backward equations, Systems Control Lett, 24, 259-263.

[22] Hamadene, S. and Wang, H. (2011) The mixed zero-sum stochastic differential game in the model with jumps. Advances in dynamic games, 83-110, Ann. Internat. Soc. Dynam. Games, 11, Birkhuser/Springer, New York, 2011.

[23] Karatzas, I. and Sudderth, W. (2006) Stochastic games of control and stopping for a linear diffusion. Random walk, sequential analysis and related topics, 100-117, World Sci. Publ., Hackensack, NJ, 2006

[24] Krylov N.K. (1987) Nonlinear elliptic and Parabolic Equations of the Second Order, Kluwer (original Russian version published in 1985).

[25] Ladyzenskaya, O.A., Solonnikov and V.A., Uralseva, N.N. (1967). Linear and Quasilinear Equations of Parabolic Type, AMS, Providence.

[26] Lieberman, G. M. Second order parabolic differential equations, World Scientific, 1998.

[27] Mou, L. and Yong, J. (2006) Two-person zero-sum linear quadratic stochastic differential games by a Hilbert space method. J. Ind. Manag. Optim. 2, no. 1, 95-117.

[28] Peng, S. (2004) Filtration consistent nonlinear expectations and evaluations of contingent claims, Acta Mathematicae Applicatae Sinica (English Series), 20, 191-214.

[29] Peng, S. (2010) Nonlinear Expectations and Stochastic Calculus under Uncertainty, Preprint, arXiv:1002.4546v1.

[30] Pham,T. and Zhang, J. (2011) Some estimates for semimartingales - Under linear and nonlinear expectations, Preprint, arXiv:1107.4020. 
[31] Soner, M., Touzi, N. and Zhang, J. (2011), Dual Formulation of Second Order Target Problems, Annals of Applied Probability, to appear, arXiv:1003.6050.

[32] Soner, M., Touzi, N. and Zhang, J. (2012), Wellposedness of Second Order BSDEs, Probability Theory and Related Fields, 153, 149-190.

[33] Stroock, D. and Varadhan, S. R. S. (1979), Multidimensional diffusion processes Springer-Verlag, New York.

[34] Swiech, A. (1996) Another Approach to the Existence of Value Functions of Stochastic Differential Games, Journal of Mathematical Analysis and Applications, 204, 884-897. 